\title{
A General Finite Volume Method for the Solution of the Reynolds Lubrication Equation with a Mass-Conserving Cavitation Model
}

\author{
Francisco J. Profito ${ }^{1,2} \cdot$ Matteo Giacopini ${ }^{3}$ - Demetrio C. Zachariadis ${ }^{1} \cdot$ \\ Daniele Dini ${ }^{2}$
}

Received: 20 June 2015/Accepted: 25 August 2015/Published online: 16 September 2015

(C) The Author(s) 2015. This article is published with open access at Springerlink.com

\begin{abstract}
This contribution presents the development of a general discretization scheme for the solution of Reynolds equation with a mass-conserving cavitation model and its application for the numerical simulation of lubricated contacts to be discretized using irregular grids. Such scheme is based on a hybrid-type formulation, here named as element-based finite volume method that combines the flexibility of the FEM to deal with unstructured grids, while preserving the local and global fluid-flow conservation aspect of the FVM throughout the discretized domain. The accuracy and robustness of the method are successfully tested using several benchmark cases proposed in the recent literature. Simulations of fully or partially textured sliding bearings are finally employed to show the advantages of being able to adopt irregular meshes both in terms of flexibility for the discretization of complex surface features and computational speed.
\end{abstract}

Keywords Lubrication - Reynolds equation - Massconserving cavitation model $\cdot$ Numerical simulations . Finite volume method

Francisco J. Profito

fprofito@hotmail.com

1 Laboratory of Surface Phenomena (LFS), Department of Mechanical Engineering, Polytechnic School of the University of São Paulo, São Paulo, Brazil

2 Department of Mechanical Engineering, Imperial College London, South Kensington Campus, London, UK

3 Dipartimento di Ingegneria "Enzo Ferrari”, Università degli Studi di Modena e Reggio Emilia, Modena, Italy

\section{Introduction}

The exact closed-form solution of the Reynolds lubrication equation exists only for very particular problems involving simple contact geometries, boundaries conditions and isothermal flows. Examples of such peculiar applications include short and long journal bearing components, as well as special formulations conceived for sliding and pure squeeze bearings. However, when the evaluation of more complicated, realistic lubrication systems is desired, approximate solutions based on numerical techniques have to be considered. The numerical solution of Reynolds equation is traditionally performed by adopting the finite difference method (FDM). The predominance of such discretization scheme is due to its relative simplicity in terms of formulation, along with the geometrically uncomplicated domains usually found in conventional lubrication systems, which may well be discretized using structured meshes. In this method, the partial derivatives of the equation are approximated by finite difference formulas obtained from the truncation of the high-order terms of Taylor expansions.

In contrast, in applications involving more complex geometries, when unstructured and/or irregular grids are necessary to accurately discretize the solution domain, the FDM has limited applicability. In the presence of irregular geometries and meshes, the finite element method (FEM) is the most widespread method employed for the numerical solution of the Reynolds equation [1-7], especially due to its great flexibility to deal with distorted elements (interpolation and shape functions). However, when mass conservation is contemplated in the fluid-film cavitation modeling by the imposition of the so-called JFO (Jakobsson, Floberg and Olsson) conditions [8-10], the solution of the modified diffusion-convection Reynolds equation 
(see, e.g., the $p-\theta$ Elrod-Adams model [11, 12]) is not straightforwardly accomplished with the FEM formulation. Essentially, the main difficulties arise in the discretization of the convective term of the modified equation, as well as in the enforcement of the flow conservation on the cavitation boundaries throughout the lubricated contact. Recently, a new FEM formulation based on the mathematical derivation of a linear complementary problem (LCP) has been proposed for solving the fluid-film lubrication with mass-conserving cavitation model [13, 14]. Moreover, [15] have developed an efficient algorithm for fluid pressure calculation defined according to a reformulation of the complementary constraints imposed by the JFO conditions.

Alternatively, the finite volume method (FVM), widely utilized in computational fluid dynamic (CFD) simulations, has been proven to be very effective for solving lubrication problem, especially in thermohydrodynamic analysis [16, 17], lubrication with discontinuous domains [18, 19] and textured surfaces [20], as well as in piston ring applications [21]. The main advantage of the FVM is its conservative characteristic, which in turn enables to impose local and global flow conservation in the discrete formulation. Such intrinsic conservative feature automatically enforces the complementary JFO conditions and allows a straightforward upwind-based treatment for the convective term of the modified Reynolds equation, hence facilitating the entire discretization procedure. The only disadvantage of conventional FVM schemes is the absence of a standardized strategy for the discretization of unstructured meshes, which is a prerogative of the FEM.

In this context, the main objective of the present contribution is to propose a new discretization scheme for solving Reynolds equation with the $p-\theta$ Elrod-Adams cavitation model on irregular meshes. Furthermore, a generalization of the cavitation algorithm proposed by Ausas [20] was proposed for application to unstructured meshes. The new scheme is based on a hybrid-type formulation, hereafter named as the element-based finite volume method (EbFVM), which combines the geometric flexibility of the FEM to deal with unstructured grids while preserving the local and global fluid-flow conservation aspect of the FVM throughout the discretized domain [22, 23]. Essentially, the EbFVM is a FVM scheme that employs the concept of elements and their interpolation and shape functions adopted in the FEM. However, different from the classical FEM methodologies, local and global conservations of the transport properties are exactly enforced in the EbFVM, since the construction of the discretized equations follows the FVM principles; this eliminates the potential issues arising from the use of the FEM mathematical foundation to derive the weak form of the formulation. In other words, in the EbFVM the discretized equations represent the physical balances over control volumes that are built as dual entities around all vertices of the primal grid, with contributions of neighboring elements. The motivation for the use of the EbFVM in lubrication applications is based on its flexibility, generality and suitability for computational implementations.

The reminder of the article is organized as follows. The mathematical formulation of the fluid-film lubrication problem (Reynolds equation) with the chosen mass-conserving cavitation model is presented in Sect. 2. The fundamentals of the EbFVM and their applications to the modified Reynolds equation are described in details in Sect. 3, where the procedure employed for the iterative solution of the discrete system of linear equations is also explained. Finally, in Sect. 4, a number of test cases based on classical examples taken from the recent literature are presented for validation purposes; the capabilities of the proposed formulation are then illustrated by simulating a textured sliding bearing to show the versatility and robustness of the proposed method to solve lubrication problems characterized by thin fluids films in the presence of cavitation, particularly when non-structured meshes are involved.

\section{Mathematical Formulations}

In this section, the mathematical formulations that describe the fluid-film lubrication problem are presented in detail. For completeness, the most general form of the equation of the mechanics of viscous thin films is considered [24, 25], as well as its modified version obtained after the consideration of the mass-conserving Elrod-Adams cavitation $(p-\theta)$ model.

\subsection{Lubrication Theory (Reynolds Equation)}

The general equation of the mechanics of viscous thin films (hereafter simply referred to as Reynolds equation) for isothermal, transient lubrication systems, in which the lubricant rheological properties (i.e., dynamic viscosity and density) are assumed constant across the film thickness, can be expressed in Cartesian coordinates as follows: 


$$
\begin{aligned}
& \underbrace{\frac{\partial}{\partial x}\left[\frac{\rho\left(H_{2}-H_{1}\right)^{3}}{12 \mu} \frac{\partial p}{\partial x}\right]+\frac{\partial}{\partial z}\left[\frac{\rho\left(H_{2}-H_{1}\right)^{3}}{12 \mu} \frac{\partial p}{\partial z}\right]}_{\text {Pressure-Flow(Poiseuille)Term }} \\
& =\underbrace{\frac{\partial}{\partial x}\left[\frac{\rho\left(U_{2}+U_{1}\right)}{2}\left(H_{2}-H_{1}\right)\right]+\frac{\partial}{\partial z}\left[\frac{\rho\left(W_{2}+W_{1}\right)}{2}\left(H_{2}-H_{1}\right)\right]}_{\text {Wedge-Flow }(\text { Couette) Term }} \\
& \underbrace{\rho\left[\left(U_{1} \frac{\partial H_{1}}{\partial x}-U_{2} \frac{\partial H_{2}}{\partial x}\right)+\left(W_{1} \frac{\partial H_{1}}{\partial z}-W_{2} \frac{\partial H_{2}}{\partial z}\right)\right]}_{\text {Translated-Squeeze Term }} \\
& +\underbrace{\rho \frac{\partial\left(H_{2}-H_{1}\right)}{\partial t}+\underbrace{\left(H_{2}-H_{1}\right) \frac{\partial \rho}{\partial t}}_{\text {Local-Expansion Term }}}_{\text {Normal-Squeeze Term }}
\end{aligned}
$$

where $p$ is the hydrodynamic pressure, $\left(H_{1}, H_{2}\right)$ the geometric descriptions of the contact surfaces and $\mu$ and $\rho$ the lubricant dynamic viscosity and density, respectively. The velocities of the two mating surfaces in the $x$ and $z$ coordinate directions are designated as $\left(U_{1}, U_{2}\right)$ and $\left(W_{1}, W_{2}\right)$, respectively; the subscripts 1 and 2 denote bottom and top surfaces according to the reference definitions in Fig. 1.

It should be noticed at this point that in Eq. 1 the normal squeeze velocities can only be produced by (local) temporal changes in the lubricant film thickness, i.e., $\left(V_{2}-V_{1}\right) \equiv \frac{\partial\left(H_{2}-H_{1}\right)}{\partial t}$. This assumption is generally valid for sliding and thrust bearings, but not necessarily for rotating-type systems as journal or rolling bearings. For the latter components, some modifications have to be taken into account in this equation, essentially by handling both the translation and normal squeeze terms in order to deal with the particular kinematic features of the systems. Such changes, however, by no means compromise the correctness and the applicability of the numerical scheme proposed in this work.

\subsection{Mass-Conserving Cavitation Model}

The cavitation (or fluid-film rupture) phenomenon plays an important role in the performance of lubrication systems and so must be included in the mathematical modeling of the problem. In order to address such cavitation effects, the mass-conserving formulation proposed by JFO (Jakobsson, Floberg and Olsson) [8-10] is here adopted. This cavitation formulation imposes proper complementary boundary conditions that ensure the mass conservation principle throughout the lubricated domain. The Elrod-Adams [11, 12] $p-\theta$ model for cavitation is utilized to automatically satisfy the JFO's conditions. In this fashion, the so-called lubricant film fraction parameter $\theta$ is introduced into the right-hand side of Eq. 1, providing the following modified $p-\theta$ version of the mechanics of viscous thin films:

$$
\begin{aligned}
& \frac{\partial}{\partial x}\left[\frac{\rho\left(H_{2}-H_{1}\right)^{3}}{12 \mu} \frac{\partial p}{\partial x}\right]+\frac{\partial}{\partial z}\left[\frac{\rho\left(H_{2}-H_{1}\right)^{3}}{12 \mu} \frac{\partial p}{\partial z}\right] \\
& =\frac{\partial}{\partial x}\left[\frac{\rho \theta\left(U_{2}+U_{1}\right)}{2}\left(H_{2}-H_{1}\right)\right]+\frac{\partial}{\partial z}\left[\frac{\rho \theta\left(W_{2}+W_{1}\right)}{2}\left(H_{2}-H_{1}\right)\right] \\
& +\rho \theta\left[\left(U_{1} \frac{\partial H_{1}}{\partial x}-U_{2} \frac{\partial H_{2}}{\partial x}\right)+\left(W_{1} \frac{\partial H_{1}}{\partial z}-W_{2} \frac{\partial H_{2}}{\partial z}\right)\right] \\
& +\rho \theta \frac{\partial\left(H_{2}-H_{1}\right)}{\partial t}+\rho\left(H_{2}-H_{1}\right) \frac{\partial \theta}{\partial t}
\end{aligned}
$$

The film fraction field $\theta$ can be interpreted as an auxiliary variable associated with the proportion of liquid (lubricant) everywhere within the domain. The magnitude of $\theta$ varies in the interval $[0,1]$, with $\theta=1$ in the pressured regions where $p \geq p_{\text {cav }}$, i.e., where the lubricant film is fully developed. However, in the cavitated zones, i.e., where $0 \leq \theta<1$, the lubricant film is broken (film rupture), and the medium is filled with a biphasic mixture of liquid and gases/vapors; the fluid pressure within these cavitation
Fig. 1 Left General schematic illustration with the coordinate system and kinematic variables defined for the derivation of the equation of the mechanics of viscous thin films. Right Detailed cross-sectional view showing the local definition of fluid-film geometry

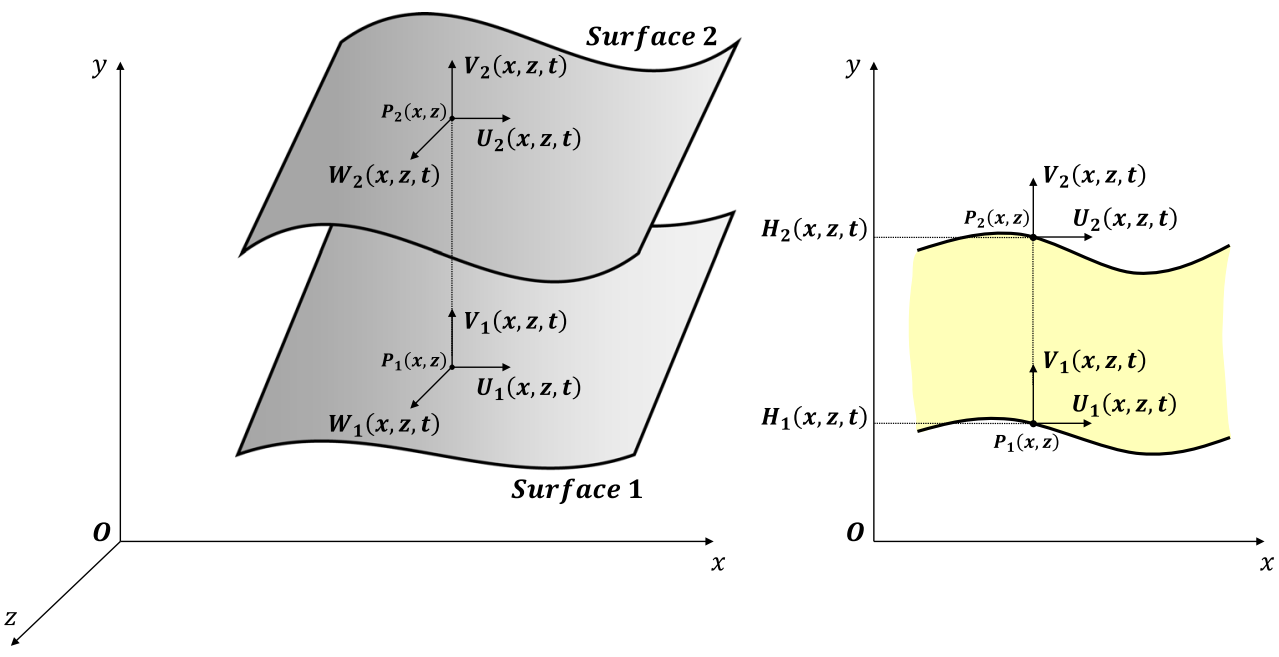


zones is assumed constant and equal to limit cavitation pressure $p_{\text {cav }}$.

\subsection{Conservative Form of the Equation of the Mechanics of Viscous Thin Films}

For the development of the EbFVM discretization scheme, it is convenient to rewrite Eq. 2 in its conservative vector form, as follows:

$\nabla \cdot\left(\boldsymbol{\Gamma}^{p} \nabla p\right)=\nabla \cdot\left(\theta \boldsymbol{\Gamma}^{c} \overrightarrow{\boldsymbol{v}}\right)+\theta\left(S_{\mathrm{TS}}+S_{\mathrm{NS}}\right)+S_{\mathrm{T}} \frac{\partial \theta}{\partial t}$

Complementary condition:

$\left(p-p_{\text {cav }}\right)(1-\theta)=0 \rightarrow\left\{\begin{array}{l}p>p_{\text {cav }} \rightarrow \theta=1 \\ p=p_{\text {cav }} \rightarrow 0 \leq \theta<1\end{array}\right.$

where $\Gamma^{p}$ and $\Gamma^{c}$ are the diffusivity and convective matrices. Moreover, the scalars $S_{\mathrm{TS}}, S_{\mathrm{NS}}$ and $S_{\mathrm{T}}$ designate the translation squeeze, normal squeeze and temporal expansion source terms. Such quantities are defined as follows:

$$
\begin{aligned}
\boldsymbol{\Gamma}^{\boldsymbol{p}} & =\left[\begin{array}{cc}
\frac{\rho\left(H_{2}-H_{1}\right)^{3}}{12 \mu} & 0 \\
0 & \frac{\rho\left(H_{2}-H_{1}\right)^{3}}{12 \mu}
\end{array}\right], \\
\boldsymbol{\Gamma}^{\boldsymbol{c}} & =\left[\begin{array}{cc}
\rho\left(H_{2}-H_{1}\right) & 0 \\
0 & \rho\left(H_{2}-H_{1}\right)
\end{array}\right] \\
S_{\mathrm{TS}} & =\rho\left(\overrightarrow{\boldsymbol{a}} \cdot \nabla H_{1}-\overrightarrow{\boldsymbol{b}} \cdot \nabla H_{2}\right), \quad S_{\mathrm{NS}}=\rho \frac{\partial\left(H_{2}-H_{1}\right)}{\partial t}, \\
S_{\mathrm{T}} & =\rho\left(H_{2}-H_{1}\right)
\end{aligned}
$$

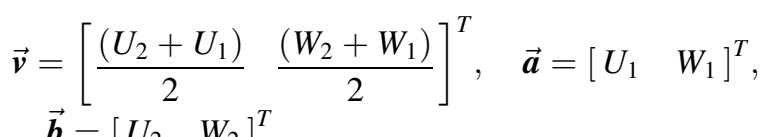

Notice that the conservative form of Eq. 3 is general, in the sense that particular geometric and kinematic characteristics of different lubrication systems, as well as possible anisotropic effects on the roughness scale in mixed lubrication applications, may be included in the formulation by easily modifying the definitions of the matrix and scalar terms of the equation.

\subsection{Lubricant Rheology}

The lubricant rheological properties, i.e., density and dynamic viscosity, are strongly affected by the temperature, pressure and shear rate conditions. Since thermal effects have been neglected in the present contribution, only the isothermal density-pressure (Dowson-Higginson equation), viscosity-pressure (e.g., Barus and/or Rhoelands equation) and viscosity-shear-thinning (Eyring equation) effects will be considered for the corrections of the lubricant properties [26, 27].

\section{Fundamentals of the Element-Based Finite Volume Method (EbFVM)}

Similar to any numerical method used to approximate the solution of partial differential equations, the initial step of the EbFVM consists in the geometric discretization of the continuum domain in smaller, simple-shaped subregiondenominated elements $(e)$, which are interconnected by nodes $(P)$ that coincide with the vertices of the elements. The collection of these geometric entities is here denominated geometric grid, and for the discretization of the Reynolds equation, it is composed by either triangular or quadrangular elements. In Fig. 2 a schematic illustration of the domain discretization and the geometric entities involved is shown.

In contrast to the classical FVM commonly used for structured grids, where elements and control volumes are coincident ("cell-center" formulation) and the transport equations are integrated directly using the geometric grid, in the EbFVM scheme the elements are used as an auxiliary entity from which the control volumes are constructed ("cell-vertex" formulation). In this case, the control volumes constitute a secondary or computational grid, where the local conservation of the physical fluxes balances are effectively enforced. As depicted in Fig. 2, a control volume $(\mathrm{CV})$ is associated to a given node $(P)$ of the geometric grid and is composed by portions of the neighboring elements. Those portions are designated as sub-control volumes (SCV) and result from a subdivision of the elements. For a barycentric subdivision (or "median rule"), the division lines are obtained by joining the centroid of the element to the midpoints of the element edges. Those lines are denoted faces $(f)$ and are the basic geometric entity from which the fluxes are locally evaluated in the discretized equations. Particularly, by assuming the "midpoint rule" to approximate the fluxes over the CV faces, the midpoint of each face (where the fluxes are effectively calculated) and its respective normal vector are defined, respectively, as integration point $(p I)$ and normal vector $(\vec{n})$. Following this strategy, each control volume associated to a given node $P$ may be seen as a sum of the nearest SCV of the elements that share $P$, and the fluxes at one specific integration point can be calculated using data from the element in which the integration point is placed [28, 29].

In contrast to the classical FVM commonly used for structured grids, where elements and control volumes are 


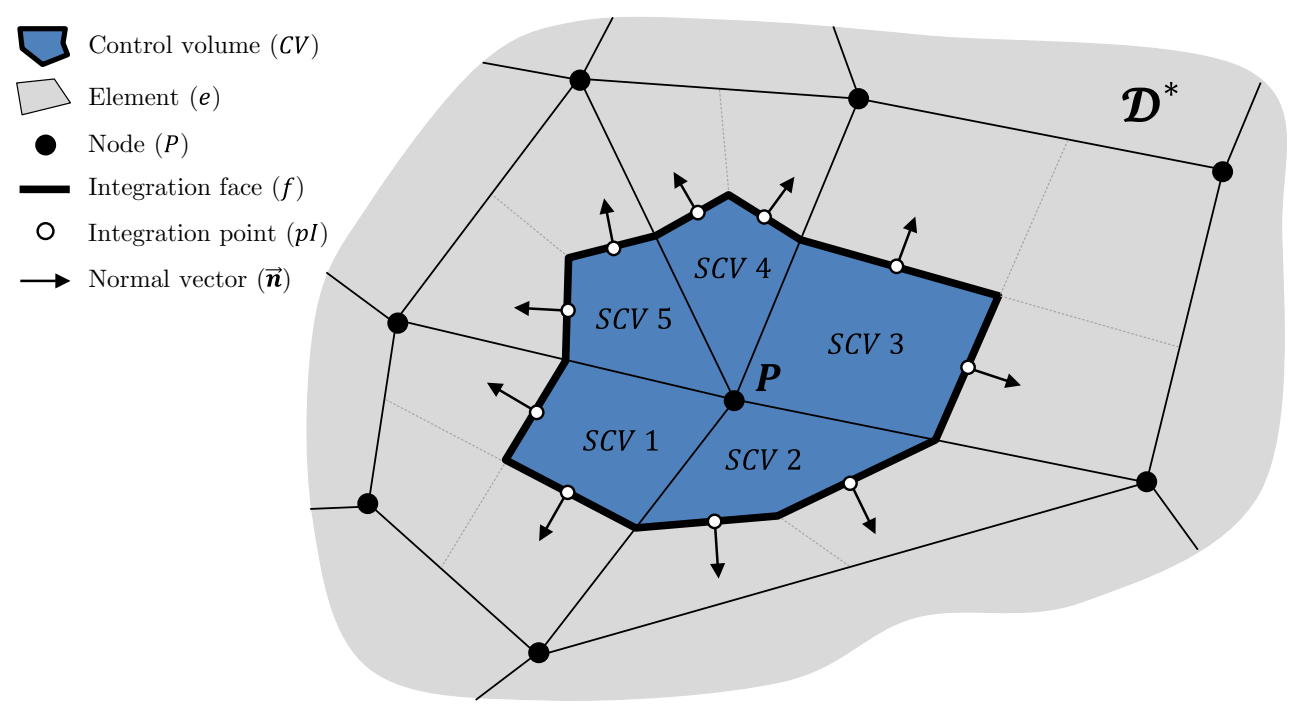

Fig. 2 Geometric entities of the element-based finite volume method (EbFVM)

coincident ("cell-center" formulation) and the transport equations are integrated directly using the geometric grid, in the EbFVM scheme the elements are used as an auxiliary entity from which the control volumes are constructed ("cell-vertex" formulation). In this case, the control volumes constitute a secondary or computational grid, where the local conservation of the physical fluxes balances are effectively enforced. As depicted in Fig. 2, a control volume $(\mathrm{CV})$ is associated to a given node $(P)$ of the geometric grid and is composed by portions of the neighboring elements. Those portions are designated as SCV and result from a subdivision of the elements. For a barycentric subdivision (or "median rule"), the division lines are obtained by joining the centroid of the element to the midpoints of the element edges. Those lines are denoted faces $(f)$ and are the basic geometric entity from which the fluxes are locally evaluated in the discretized equations. Particularly, by assuming the "midpoint rule" to approximate the fluxes over the $\mathrm{CV}$ faces, the midpoint of each face (where the fluxes are effectively calculated) and its respective normal vector are defined, respectively, as integration point $(p I)$ and normal vector $(\overrightarrow{\boldsymbol{n}})$. Following this strategy, each control volume associated to a given node $P$ may be seen as a sum of the nearest $\mathrm{SCV}$ of the elements that share $P$, and the fluxes at one specific integration point can be calculated using data from the element in which the integration point is placed [28, 29].

As already stated, due to the "cell-vertex" nature of the EbFVM, the unknowns of the problem are approximated at the nodes of the geometric grid. Furthermore, in order to deal with the distorted elements of irregular grids, as well as local variations in the flow and transport properties, predefined families of interpolation and shape functions can be employed. These functions are based on the definition of parametric elements that are described with respect to a local (transformed) coordinate system. Thus, all the required calculations previously undertaken in the physical domain can be performed more easily in the transformed domain. The reader should refer to Appendix 1 for details about the coordinate transformation and the associated shape functions of triangular and quadrangular elements.

Once the integration points are identified within each element, the fluxes of the transport properties through the faces of the SCV can be calculated according to the geometric and general parameters associated with the element nodes. This procedure allows elements, and so the geometric grid, to be treated as the major entity over which all the calculations are performed independently. Subsequently, the conservation equation for every control volume can be determined from the fluxes contributions coming from its respective communal surrounding SCV. Thus, after an element-by-element assembly of the global system of equations (analogous to the assembly procedure in the FEM), the total physical balance of the conservation equation is automatically satisfied by the inherent connectivity of the elements.

In the next sections, the temporal and spatial discretization of each term of the modified Reynolds equation (Eq. 3a) will be presented in details. Further explanations concerning the EbFVM scheme for a generic conservation equation can be found in $[22,23,28]$.

\subsection{Temporal Discretization}

The temporal approximation of Eq. 3a can be established by integrating all terms of the equation in a time interval $\Delta t$, as follows: 


$$
\begin{aligned}
\int_{t}^{t+\Delta t}\left[\nabla \cdot\left(\boldsymbol{\Gamma}^{p} \nabla p\right)\right] \mathrm{d} t= & \int_{t}^{t+\Delta t}\left[\nabla \cdot\left(\theta \boldsymbol{\Gamma}^{c} \overrightarrow{\boldsymbol{v}}\right)\right] \mathrm{d} t \\
& +\int_{t}^{t+\Delta t}\left[\theta\left(\mathrm{S}_{\mathrm{TS}}+\mathrm{S}_{\mathrm{NS}}\right)\right] \mathrm{d} t \\
& +\int_{t}^{t+\Delta t}\left[S_{\mathrm{T}} \frac{\partial \theta}{\partial t}\right] \mathrm{d} t
\end{aligned}
$$

Assuming an implicit first-order time discretization scheme (backward Euler method), the integrands of Eq. 5 can be approximated to their values at the end of the time interval. The advantage of such formulation is its unconditional stability, so that the numerical accuracy of the solution is mainly controlled by the size of the time step $\Delta t$. Thus, by performing the temporal discretization using $t=n \Delta t$, where $n$ is the $n$-th time instant, Eq. 5 can be rewritten as:

$$
\begin{aligned}
{\left[\nabla \cdot\left(\boldsymbol{\Gamma}^{p} \nabla p\right)\right]^{n}=} & {\left[\nabla \cdot\left(\theta \boldsymbol{\Gamma}^{c} \overrightarrow{\boldsymbol{v}}\right)\right]^{n}+\left[\theta\left(S_{\mathrm{TS}}+S_{\mathrm{NS}}\right)\right]^{n} } \\
& +S_{\mathrm{T}}^{n}\left(\frac{\theta^{n}-\theta^{n-1}}{\Delta t}\right)
\end{aligned}
$$

\subsection{Spatial Discretization}

For the spatial discretization of Eq. 6 at each time instant $t=n \Delta t$, it is convenient to consider its integral formulation (or weak formulation), which may be obtained by adopting the weighted residual method (WRM). The reason for using such integral approach is to ensure, mathematically, the minimization of the approximation errors throughout the solution domain [30]. In this case, Eq. 6 is expressed as follows:

$$
\begin{aligned}
& \iint_{\mathcal{D}^{*}} \mathcal{W}\left\{\left[\nabla \cdot\left(\tilde{\boldsymbol{\Gamma}}^{p} \nabla \tilde{p}\right)^{n}\right]-\left[\nabla \cdot\left(\tilde{\theta} \tilde{\boldsymbol{\Gamma}}^{c} \tilde{\overrightarrow{\boldsymbol{v}}}\right)^{n}\right]\right. \\
& \left.\quad-\left[\tilde{\theta}\left(\tilde{S}_{\mathrm{TS}}+\tilde{S}_{\mathrm{NS}}\right)\right]^{n}-\tilde{S}_{\mathrm{T}}^{n}\left(\frac{\tilde{\theta}^{n}-\tilde{\theta}^{n-1}}{\Delta t}\right)\right\} \mathrm{d} \mathcal{D}^{*}=0
\end{aligned}
$$

where $\mathcal{W}$ corresponds to the weighting function that weights the discretization errors over the entire integration domain $\left(\mathcal{D}^{*}\right)$, and the superscript ' $\sim$ ' denotes the approximated solutions of the transport properties and unknown variables. Particularly for FVMs, $\mathcal{W}$ is assumed unitary within every control volume and null outside (subdomain method) [31]. Thus, Eq. 7 can be restated for each control volume as:

$$
\begin{aligned}
\iint_{\mathcal{D}}\left[\nabla \cdot\left(\tilde{\boldsymbol{\Gamma}}^{p} \nabla \tilde{p}\right)^{n}\right] \mathrm{d} \mathcal{D}= & \iint_{\mathcal{D}}\left[\nabla \cdot\left(\tilde{\theta} \tilde{\boldsymbol{\Gamma}}^{c} \tilde{\overrightarrow{\boldsymbol{v}}}\right)^{n}\right] \mathrm{d} \mathcal{D} \\
& +\iint_{\mathcal{D}}\left[\tilde{\theta}\left(\tilde{S}_{\mathrm{TS}}+\tilde{S}_{\mathrm{NS}}\right)\right]^{n} \mathrm{~d} \mathcal{D} \\
& +\iint_{\mathcal{D}}\left[\tilde{S}_{\mathrm{T}}^{n}\left(\frac{\tilde{\theta}^{n}-\tilde{\theta}^{n-1}}{\Delta t}\right)\right] \mathrm{d} \mathcal{D}
\end{aligned}
$$

where $\mathcal{D}$ is the sub-domain enclosed by each control volume centered at every node $P$.

By applying the divergence (or Gauss-Ostrogradsky's) theorem to the first two integrals of Eq. 8, one obtains:

$$
\begin{aligned}
\oint_{\mathcal{C}}\left[\left(\tilde{\boldsymbol{\Gamma}}^{p} \nabla \tilde{\boldsymbol{p}}\right)^{n} \cdot \overrightarrow{\boldsymbol{n}}\right] \mathrm{d} \mathcal{C}= & \oint_{\mathcal{C}}\left[\left(\tilde{\theta} \tilde{\boldsymbol{\Gamma}}^{c} \tilde{\overrightarrow{\boldsymbol{v}}}\right)^{n} \cdot \overrightarrow{\boldsymbol{n}}\right] \mathrm{d} \mathcal{C} \\
& +\iint_{\mathcal{D}}\left[\tilde{\theta}\left(\tilde{S}_{\mathrm{TS}}+\tilde{S}_{\mathrm{NS}}\right)\right]^{n} \mathrm{~d} \mathcal{D} \\
& +\iint_{\mathcal{D}}\left[\tilde{S}_{\mathrm{T}}^{n}\left(\frac{\tilde{\theta}^{n}-\tilde{\theta}^{n-1}}{\Delta t}\right)\right] \mathrm{d} \mathcal{D}
\end{aligned}
$$

where $\mathcal{C}$ is the boundary that delimits each control volume and $\overrightarrow{\boldsymbol{n}}$ the unit vector normal to that boundary (see Fig. 2).

Finally, recalling that the fluxes over the CV faces are approximated by the "midpoint rule" and by assuming a second-order approximation scheme for the area integrals (in which the integral is evaluated as the product between the integrant function at the CV center and the CV area [32]), Eq. 9 can be expressed in its discretized form as follows:

$$
\begin{aligned}
& \sum_{e \in \mathcal{E}_{P} f \in \mathcal{F}_{P}^{e}}\left[\left(\tilde{\boldsymbol{\Gamma}}^{p} \nabla \tilde{p}\right)^{n} \cdot \overrightarrow{\boldsymbol{n}}^{*}\right]_{f}^{e}=\sum_{e \in \mathcal{E}_{P}} \sum_{f \in \mathcal{F}_{P}^{e}}\left[\left(\tilde{\theta} \tilde{\boldsymbol{\Gamma}}^{c} \tilde{\vec{v}}\right)^{n} \cdot \overrightarrow{\boldsymbol{n}}^{*}\right]_{f}^{e} \\
& +\left[\tilde{\theta}\left(\tilde{S}_{\mathrm{TS}}+\tilde{S}_{\mathrm{NS}}+\frac{\tilde{S}_{\mathrm{T}}}{\Delta t}\right)\right]_{P}^{n} \underbrace{\sum_{e \in \mathcal{E}_{P}}\left[\Delta \mathcal{D}_{s}^{e}\right]}_{\Delta \mathcal{D}_{P}} \\
& -\left[\tilde{\theta}^{n-1}\left(\frac{\tilde{S}_{\mathrm{T}}}{\Delta t}\right)^{n}\right] \underbrace{\sum_{e \in \mathcal{E}_{P}}\left[\Delta \mathcal{D}_{s}^{e}\right]}_{P}
\end{aligned}
$$

where $\overrightarrow{\boldsymbol{n}}_{f}^{* e}=\overrightarrow{\boldsymbol{n}}_{f}^{e} \Delta \mathcal{C}_{f}^{e}$ is the normal face vector that retains, simultaneously, the normal orientation $\left(\overrightarrow{\boldsymbol{n}}_{f}^{e}\right)$ and the length $\left(\Delta \mathcal{C}_{f}^{e}\right)$ of each delimiting face associated with a given control volume; $\Delta \mathcal{D}_{s}^{e}$ is the area of the $s$-th SCV that compound the control volume and $\Delta \mathcal{D}_{P}$ is the full area of the respective $\mathrm{CV}$ (see Appendix 1 for details about the computation of $\overrightarrow{\boldsymbol{n}}_{f}^{* e}$ for triangular and quadrangular elements). Moreover, in Eq. $10 \mathcal{E}_{P}$ denotes the set of elements that contribute to the formation of the $\mathrm{CV}$ around node $P$ and $\mathcal{F}_{P}^{e}$ the set of faces inside the corresponding element $e$ that encloses $P$.

The above discrete equation is valid for every control volume constructed surrounding each node $P$ of the geometry grid. The inner summations in the first two terms of the equation correspond, respectively, to the diffusive and convective fluxes through each sub-control volume, while the outer summations represent the entire flux balances over the associated CV. The last two components of the equation are the source terms evaluated explicitly at node $P$. 
As already pointed out, the assembly of the global system of equations is performed in an element-by-element fashion, similar to the procedure employed in the FEM. Furthermore, all local computations are carried out at element level using the transformed domain approach (parametric elements); this allows the elements to be treated independently, no matter how distorted they are with respect to the global coordinate system. Such procedure renders the definition of element matrices and vectors constituting each term of Eq. 10 straightforward, analogously to the stiffness and mass matrices and load vectors used in FEM formulations. In the following, those definitions will be exposed in details by adopting the index notation for compactness.

\subsubsection{Diffusivity Matrix (Poiseuille Term)}

The total diffusive flux (first term of Eq. 10) across the $\mathrm{SCV}$ of a given element $e$ can be represented as:

$\overrightarrow{\boldsymbol{d}}_{e}^{n}=\boldsymbol{P}_{e}^{n}(\overrightarrow{\tilde{\boldsymbol{p}}})_{e}^{n}$

where the components of the vector $\overrightarrow{\boldsymbol{d}}_{e}^{n}$ correspond to the diffusive fluxes through each sub-control volume, $(\overrightarrow{\tilde{\boldsymbol{p}}})_{e}^{n}$ is the vector of the nodal pressures and $\boldsymbol{P}_{e}^{n}$ is the diffusivity matrix of the element. See Appendix 2 for details about the derivation of $\boldsymbol{P}_{e}^{n}$.

\subsubsection{Convective Matrix (Couette Term)}

Similar to the diffusion term, the total convective flux (second term of Eq. 10) flowing through the SCV of a given element $e$ can be expressed as:

$\overrightarrow{\boldsymbol{c}}_{e}^{n}=\boldsymbol{C}_{e}^{n}(\overrightarrow{\tilde{\boldsymbol{\theta}}})_{e}^{n}$

where the entries of the vector $\overrightarrow{\boldsymbol{c}}_{e}^{n}$ are the convective fluxes through each sub-control volume, $(\overrightarrow{\tilde{\boldsymbol{\theta}}})_{e}^{n}$ the vector of the nodal lubricant film fraction, and $\boldsymbol{C}_{e}^{n}$ is the convective matrix of the element. See Appendix 3 for details about the derivation of $\boldsymbol{C}_{e}^{n}$.

\subsubsection{Source Term Vectors}

The source terms (third and fourth terms of Eq. 10) associated with the SCV of a given element $e$ can be written as follows:

$$
\begin{aligned}
& \left(\overrightarrow{\boldsymbol{f}}_{1}\right)_{e}^{n}=\left(\overrightarrow{\boldsymbol{F}}_{1}\right)_{e}^{n} \cdot(\overrightarrow{\tilde{\boldsymbol{\theta}}})_{e}^{n} \\
& \left(\overrightarrow{\boldsymbol{f}}_{2}\right)_{e}^{n}=\left(\overrightarrow{\boldsymbol{F}}_{2}\right)_{e}^{n} \cdot(\overrightarrow{\tilde{\boldsymbol{\theta}}})_{e}^{n-1}
\end{aligned}
$$

where the members of the vectors $\left(\vec{f}_{1}\right)_{e}^{n}$ and $\left(\vec{f}_{2}\right)_{e}^{n}$ are the source terms of each element node, while $\left(\overrightarrow{\boldsymbol{F}}_{1}\right)_{e}^{n}$ and $\left(\overrightarrow{\boldsymbol{F}}_{2}\right)_{e}^{n}$ are auxiliaries element vectors (see Appendix 4 for more details about their derivations). Additionally, $(\overrightarrow{\tilde{\boldsymbol{\theta}}})_{e}^{n}$ and $(\tilde{\tilde{\boldsymbol{\theta}}})_{e}^{n-1}$ are the vectors of the nodal lubricant film fraction at the time instants $n$ and $(n-1)$, respectively. In the above equations, the operator "." denotes scalar product.

\subsection{Assembly of the Global Linear System of Equations}

Subsequently to the calculation of the diffusivity and convective matrices, as well as the source term vectors for all elements of the geometric grid, the discrete equation associated with every control volume are assembled in a global system of linear equations. The rows of the resulting linear system correspond to the entire flux balance over each control volume constructed around every node $P$ of the grid. This can be mathematically represented as:

$$
\begin{aligned}
\left(a_{P}^{n}\right) \tilde{p}_{P}^{n}+\sum_{\mathrm{NB}}\left[\left(a_{\mathrm{NB}}^{n}\right) \tilde{p}_{\mathrm{NB}}^{n}\right]= & \left(b_{P}^{n}\right) \tilde{\theta}_{P}^{n}+\sum_{\mathrm{NB}}\left[\left(b_{\mathrm{NB}}^{n}\right) \tilde{\theta}_{\mathrm{NB}}^{n}\right] \\
& +B_{P}^{n}
\end{aligned}
$$

where NB denotes the neighboring nodes around $P$ and the coefficients $a_{i}^{n}$ and $b_{i}^{n}$ are the entries of the linear system of equations computed during the assembly operation.

\subsection{Solution of the Linear System of Equations}

For each time instant $n$, the global system of equations defined in the previous section (Eq. 14), embraces two unknown variables, namely the hydrodynamic pressure $(\tilde{p})$, and the lubricant film fraction $(\tilde{\theta})$ fields. Thus, a specific numerical procedure has to be considered for the simultaneous solution of $\tilde{p}$ and $\tilde{\theta}$. In the present contribution, such simultaneous calculations are accomplished by adopting a generalization of the cavitation algorithm proposed by [20], which was originally conceived for applications with structured grids. In this sense, the generalization here devised consists in an extension of the original algorithm for lubrication problems to be discretized by irregular meshes.

The main idea of the algorithm is based on the iterative solution of linear system of equations according to the well-known Gauss-Seidel method with successive overrelaxation (SOR). In this case, by assuming that $a_{i}^{n}, b_{i}^{n}$ and $B_{i}^{n}$ are known a priori, the nodal values of $\tilde{p}_{P}^{n}$ and $\tilde{\theta}_{P}^{n}$ can be computed iteratively, as follows:

$$
\begin{aligned}
\left(\tilde{p}_{P}^{n}, \tilde{\theta}_{P}^{n}\right) & =\lim _{r \rightarrow \infty}\left(\tilde{p}_{P}^{n, r}, \tilde{\theta}_{P}^{n, r}\right) \text { with }\left(\tilde{p}_{P}^{n, 0}, \tilde{\theta}_{P}^{n, 0}\right) \\
& =\left(\tilde{p}_{P}^{n-1}, \tilde{\theta}_{P}^{n-1}\right)
\end{aligned}
$$


$\tilde{p}_{P}^{n, r}=\omega_{p} \tilde{p}_{P}^{n, r}+\left(1-\omega_{p}\right) \tilde{p}_{P}^{n, r-1}$

$\tilde{\theta}_{P}^{n, r}=\omega_{\theta} \tilde{\Theta}_{P}^{n, r}+\left(1-\omega_{\theta}\right) \tilde{\theta}_{P}^{n, r-1}$

where $r$ is the $r$-th iteration of the SOR method, $\omega_{p}$ and $\omega_{\theta}$ the respective relaxation factors, and $\tilde{P}_{P}^{n, r}$ and $\tilde{\Theta}_{P}^{n, r}$ the intermediate nodal values of $\tilde{p}$ and $\tilde{\theta}$.

The intermediate quantities $\tilde{P}_{P}^{n, r}$ and $\tilde{\Theta}_{P}^{n, r}$ are approximated from Eq. 14 by isolating the respective nodal values and admitting the others fixed to the values calculated in the previous iteration, which mathematically corresponds to:

$$
\begin{aligned}
\tilde{P}_{P}^{n, r}= & \frac{1}{a_{P}^{n}}\left\{-\sum_{\mathrm{NB}}\left[\left(a_{\mathrm{NB}}^{n}\right) \tilde{P}_{\mathrm{NB}}^{n, r-1}\right]+\left(b_{P}^{n}\right) \tilde{\theta}_{P}^{n, r-1}\right. \\
& \left.+\sum_{\mathrm{NB}}\left[\left(b_{\mathrm{NB}}^{n}\right) \tilde{\theta}_{\mathrm{NB}}^{n, r-1}\right]+B_{P}^{n}\right\}, \\
\tilde{\Theta}_{P}^{n, r}= & \frac{1}{b_{P}^{n}}\left\{\left(a_{P}^{n}\right) \tilde{p}_{P}^{n, r-1}+\sum_{\mathrm{NB}}\left[\left(a_{\mathrm{NB}}^{n}\right) \tilde{p}_{\mathrm{NB}}^{n, r-1}\right]\right. \\
& \left.-\sum_{\mathrm{NB}}\left[\left(b_{\mathrm{NB}}^{n}\right) \tilde{\theta}_{\mathrm{NB}}^{n, r-1}\right]-B_{P}^{n}\right\} .
\end{aligned}
$$

Furthermore, the complementary conditions related to the $p-\theta$ cavitation model displayed in Eq. $3 \mathrm{~b}$ must be satisfied during the iterative procedure. In the discrete domain this reads:

$\left\{\begin{array}{l}\tilde{p}_{P}^{n, r}>p_{\mathrm{cav}} \\ 0 \leq \tilde{\theta}_{P}^{n, r} \leq 1\end{array} \Rightarrow\left\{\begin{array}{l}\tilde{p}_{P}^{n, r} \geq p_{\mathrm{cav}} \rightarrow \tilde{\theta}_{P}^{n, r}=1 \\ \tilde{\theta}_{P}^{n, r}<1 \rightarrow \tilde{p}_{P}^{n, r}=p_{\mathrm{cav}}\end{array}\right.\right.$.

The discrete complementary conditions of Eq. 17 enforce the cavitation regions and boundaries to be positioned always at the nodes of the geometric grid. Moreover, as the discretization method is inherently conservative in the discrete domain (finite volume method), the local conservation of the lubricant flow is ensured locally for all grid nodes (control volumes), including those laying on the cavitation boundaries; this automatically satisfies the mass conservation conditions imposed by the JFO cavitation model. The extended SOR algorithm proposed in this contribution is shown as a pseudo-code in Appendix 5.

\section{Results and Discussion}

In this section, the results of several simulations cases performed using the EbFVM discretization scheme and the iterative algorithm described above are presented. Initially, examples from the literature have been considered for validation purposes, followed by simulations of a textured sliding bearing with different dimples' distribution aimed to illustrate the flexibility of the method for dealing with irregular meshes.

\subsection{Validation Cases}

The example cases considered in this section have been chosen to test the correctness and accuracy of the proposed formulation and algorithmic implementation by comparison with other solution methodologies published in the recent literature, including both alternative mass-conserving Reynolds' solvers and full CFD simulations. Each considered example has been selected to demonstrate the various features considered in the newly developed computational framework. It should be emphasized here that the main focus of the work presented by the authors is the validation of the newly developed discretization scheme rather than the direct comparison of performance between different algorithms proposed in the literature.

- Single and double parabolic slider with density-pressure correction

This first example has been chosen to evaluate the effectiveness of the proposed EbFVM formulation in comparison with different methodologies for solving lubrication problems involving compressible fluids. To do so, two simulation cases characterized by single and double parabolic sliders similar to those tested in [33] have been analyzed. However, as proposed in [14], the lubricant film geometry has been modified with respect to that used in [33] in order to highlight the effects of the fluid compressibility. The main geometric and operational parameters of each parabolic slider are summarized in Table 1. The results for the single parabolic slider are examined against those calculated using the formulations proposed in [33], the LCP solution in [14] and the incompressible case. Figure 3a confirms the agreement of the current EbFVM formulation with those obtained with the algorithms proposed by Sahlin et al. [33] and Bertocchi et al. [14], both in terms of hydrodynamic pressures (maximum difference in peak pressure of $0.76 \%$ ) and location of the cavitation boundary separating the pressured and cavitated zones.

Additionally, the double parabolic slider case also proposed by [33] is used to assess the capability of the EbFVM for predicting the fluid-film reformation boundary. The results are compared both with respect to a CFD analysis based on the full solution of the Navier-Stokes equations developed using the OpenFOAM code [34], as well as with the same methods employed by Sahlin et al. [33] and Bertocchi et al. [14]. The consideration of CFD solutions as a comparative basis is aimed to (1) ensure the validity of Reynolds 
Table 1 General simulation parameters for the single and double parabolic slider cases with density-pressure correction

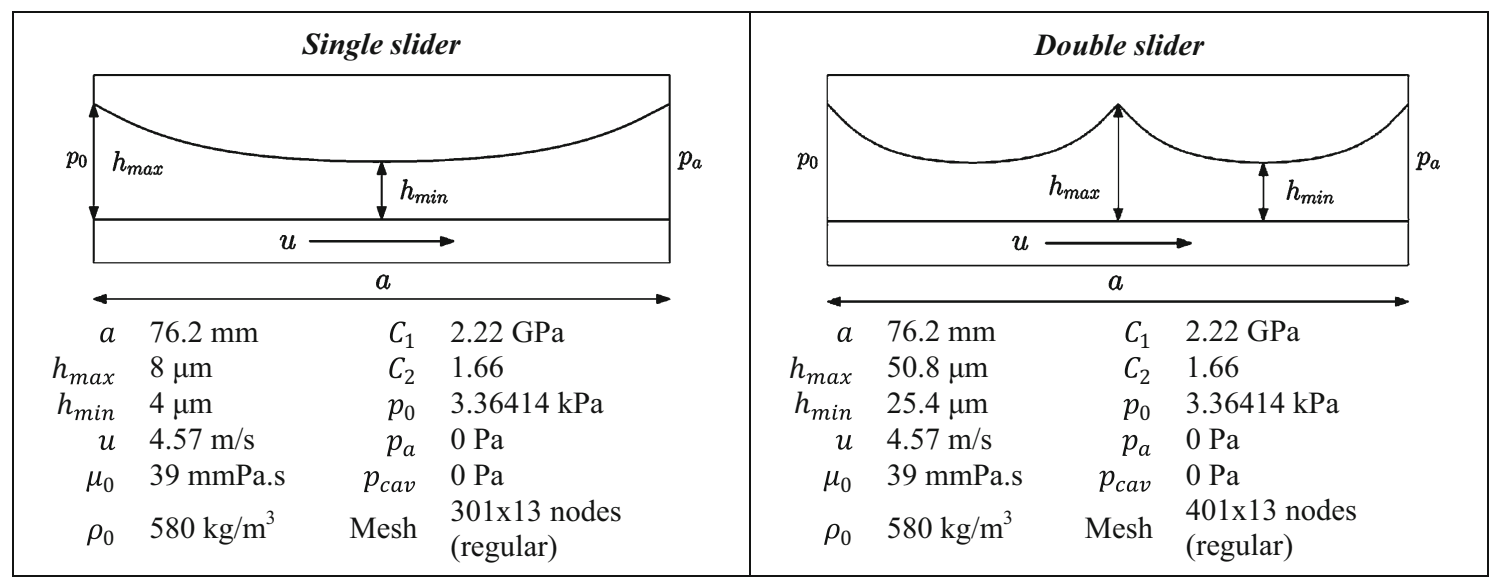

Fig. 3 Comparative results for the single (a) and double (b) parabolic slider cases with density-pressure correction
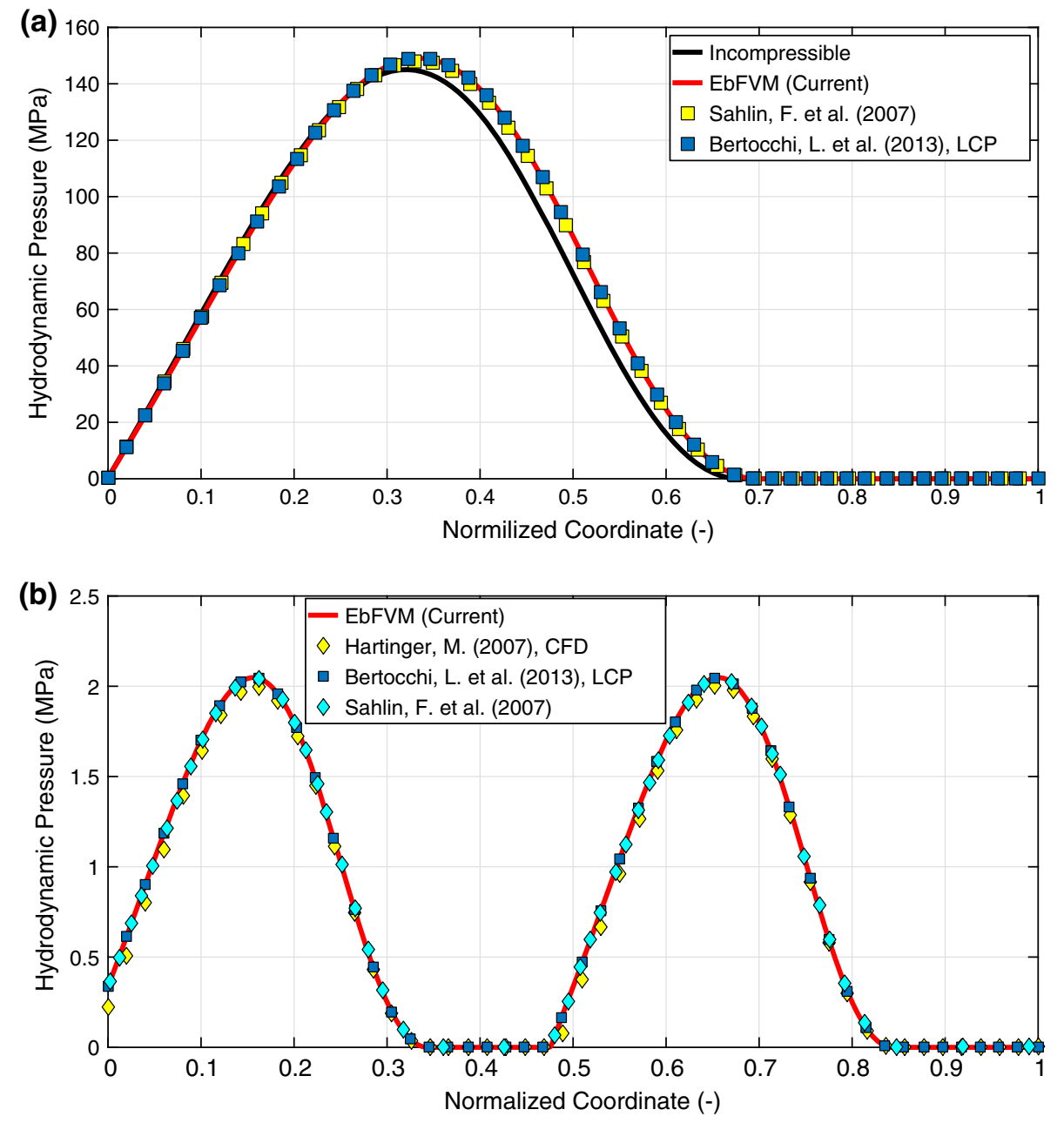

equation for flow problems which satisfy the fundamental lubrication hypotheses, and further (2) demonstrate that in common lubrication applications the intricate flow behavior of the liquid-vapor/gases mixture within the cavitation zones is in general meaningless for the overall predictions of the system performance. Figure $3 \mathrm{~b}$ illustrates an excellent agreement between the results calculated with the current 
EbFVM scheme compared to those obtained from the CFD simulations and using the methodologies by Sahlin et al. [33] and Bertocchi et al. [14]; the maximum deviation for the hydrodynamic peak

Table 2 General simulation parameters for the infinitely long journal bearing case with pressure-viscosity correction

\begin{tabular}{ll}
\hline$R$ & $31.29 \mathrm{~mm}$ \\
$L$ & $625.8 \mathrm{~mm}$ \\
$c$ & $40.0 \mu \mathrm{m}$ \\
$\omega$ & $250 \mathrm{rad} / \mathrm{s}$ \\
$\varepsilon$ & $0.93 \mathrm{and} 0.95$ \\
$\mu_{0}$ & $5.7 \mathrm{~mm} \mathrm{~Pa} \mathrm{~s}$ \\
$\rho_{0}$ & $850 \mathrm{~kg} / \mathrm{m}^{3}$ \\
$\alpha$ & $11.2 \mathrm{GPa}^{-1}$ \\
$p_{0}$ & $0 \mathrm{~Pa}$ \\
$p_{\text {cav }}$ & $-100 \mathrm{kPa}$ \\
Mesh & $601 \times 13$ nodes (regular) \\
\hline
\end{tabular}

pressure is $\leq 0.5 \%$ and the rupture and reformation cavitation boundaries are practically identical.

- Long journal bearing with viscosity-pressure correction In this second example, the capability of the proposed EbFVM formulation for solving problems with piezoviscous lubricants is tested. Particularly, the simulation case of an infinitely long journal bearing solved in [14] based on the LCP approach for an incompressible piezo-viscous lubricant flow is considered. The main problem parameters are listed in Table 2. Two different operational conditions represented by the fixed eccentricity ratios of $\varepsilon=0.93$ and $\varepsilon=0.95$ are investigated. The Barus equation is adopted for the piezo-viscosity correction. Figure 4 compares the hydrodynamic pressure profiles along the bearing circumferential directions computed with the current EbFVM formulation against the results obtaining using the LCP method [14] for the two eccentricity ratios considered. Again, the results are in good agreement in both cases, with a maximum peak pressure difference of $0.9 \%$. Excellent
Fig. 4 Comparative results for the infinitely long journal bearing case with pressureviscosity correction. a $\varepsilon=0.93$ and $\mathbf{b} \varepsilon=0.95$
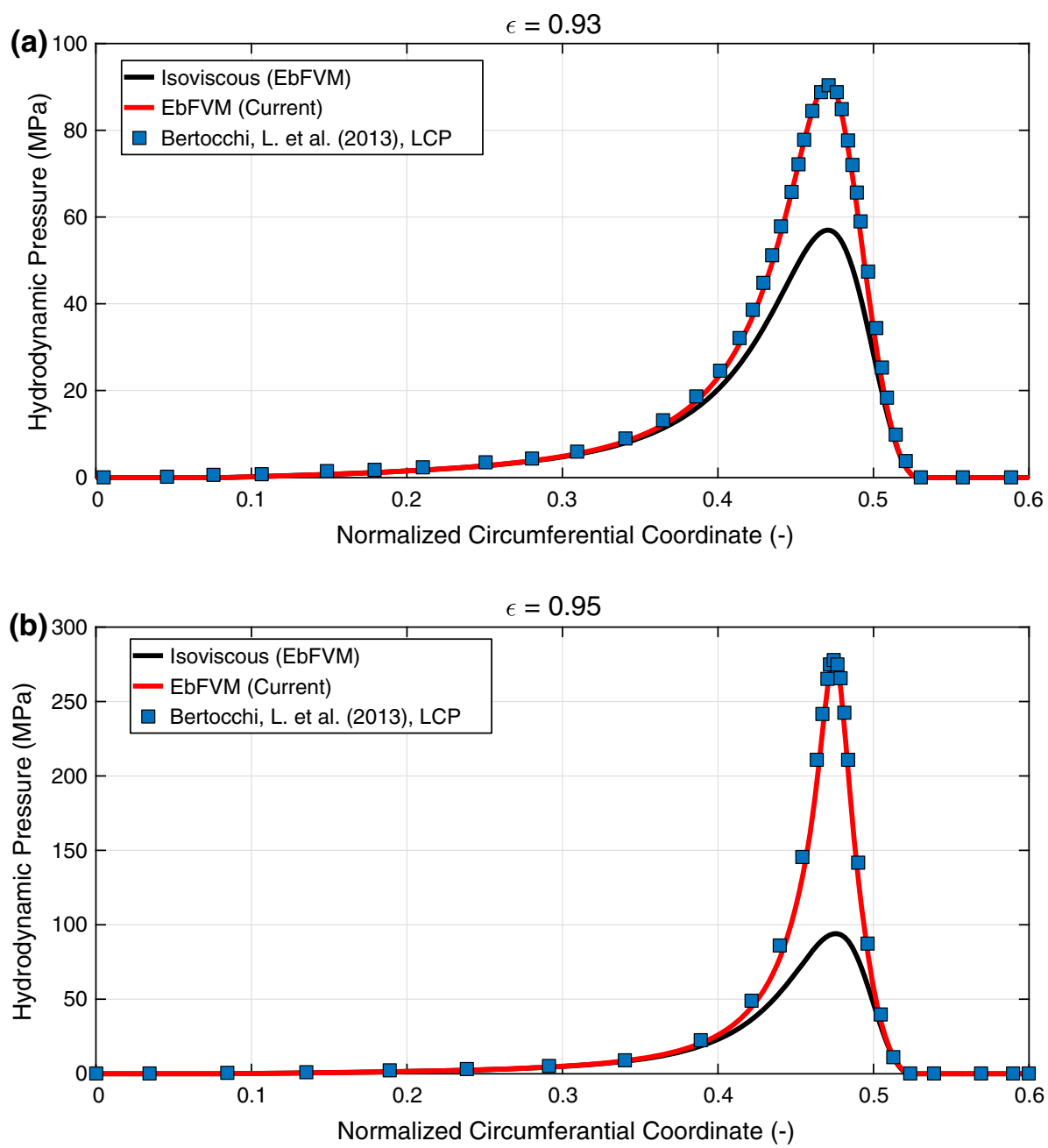
Table 3 General simulation parameters for the pure squeeze motion case between circular plates

\begin{tabular}{ll}
\hline$r$ & $5 \mathrm{~mm}$ (circular) \\
$h(t)$ & $h_{\min }+h_{a}(1-\cos \omega t)$ \\
$h_{\min }$ & $9.14 \mathrm{~mm}$ \\
$h_{a}$ & $320.8 \mu \mathrm{m}$ \\
$t_{\max }$ & $0.1890 \mathrm{~s}(3$ cycles $)$ \\
$n_{t}$ & $576($ per cycle $)$ \\
$\omega$ & $99.74 \mathrm{rad} / \mathrm{s}$ \\
$T$ & $0.063 \mathrm{~s}$ \\
$\mu_{0}$ & $5 \mathrm{~mm} \mathrm{~Pa} \mathrm{~s}$ \\
$\rho_{0}$ & $850 \mathrm{~kg} / \mathrm{m}^{3}$ \\
$p_{0}$ & $100 \mathrm{kPa}$ \\
Mesh & $2913 \mathrm{nodes}$ (irregular) \\
\hline
\end{tabular}

agreement is observed for the cavitation boundaries as well.

- Pure squeeze motion between circular plates

In this example case, the validation of the proposed EbFVM scheme is assessed for a two-dimensional domain under time-dependent conditions. The classical lubrication problem of two circular plates with finite radius and under pure (sinusoidal) squeeze motion is considered. Moreover, any viscosity and density corrections are ignored. The results are compared with those obtained from the analytical solution developed by [3] and the linear complementary method of [14]. The main system parameters are displayed in Table 3. For the numerical simulation, an irregular grid with 2913 nodes has been used, and three oscillating periods with 576 times steps per cycle are adopted for the time solution. Figure 5 depicts the time variation of the extension of the cavitation zone calculated from the above-described methodologies chosen for comparison; notice that the internal "annulus-like" surface plots correspond to the simulated film fraction fields at three different times, which illustrate the progressive variation of the cavitation zone (in blue). Once again, the agreement of the results is excellent.

- Sliding pocket bearing with viscosity-pressure, viscosity-shear-thinning and density-pressure correction

This second two-dimensional example deals with a sliding pocket bearing lubricated with a compressible, piezo-vscous and shear-thinning fluid. Two geometric configurations characterizing a finite and an infinitely long bearing are investigated. All the geometric and operational parameters are listed in Table 4. Figure 6 compares the pressure profiles along the bearing midsection $\pi$ calculated with the current EbFVM scheme and with the LCP formulation by Bertocchi et al. [14]. As can be observed, the results computed with the new finite volume scheme match very well those obtained with LCP technique for both bearing geometries, including the analytical solution for the infinitely long bearing case.

\subsection{Textured Sliding Bearing Simulation}

In order to illustrate the robustness and flexibility of the EbFVM to tackle irregular and dense meshes, a set of simulations for a parallel and flat textured sliding bearing have been undertaken for the geometric and operational parameters listed in Table 5. Full and partial texturing are considered by varying the number of dimples' cells added onto the stationary bearing surface from $10 \times 2$ to $10 \times 10$ cells; the dimples are spherical in shape and cover approximately $20 \%$ of each texture cell (see details of the dimples' dimensions in Table 5). Moreover, the lubricant density and viscosity are assumed constant as well.

The lubricated domains are discretized using both regular and irregular grids; in particular, the latter grid type is used for allowing mesh refinements in the neighborhoods of the texture features, where the pressure gradients are
Fig. 5 Comparative results for the pure squeeze motion case between circular plates

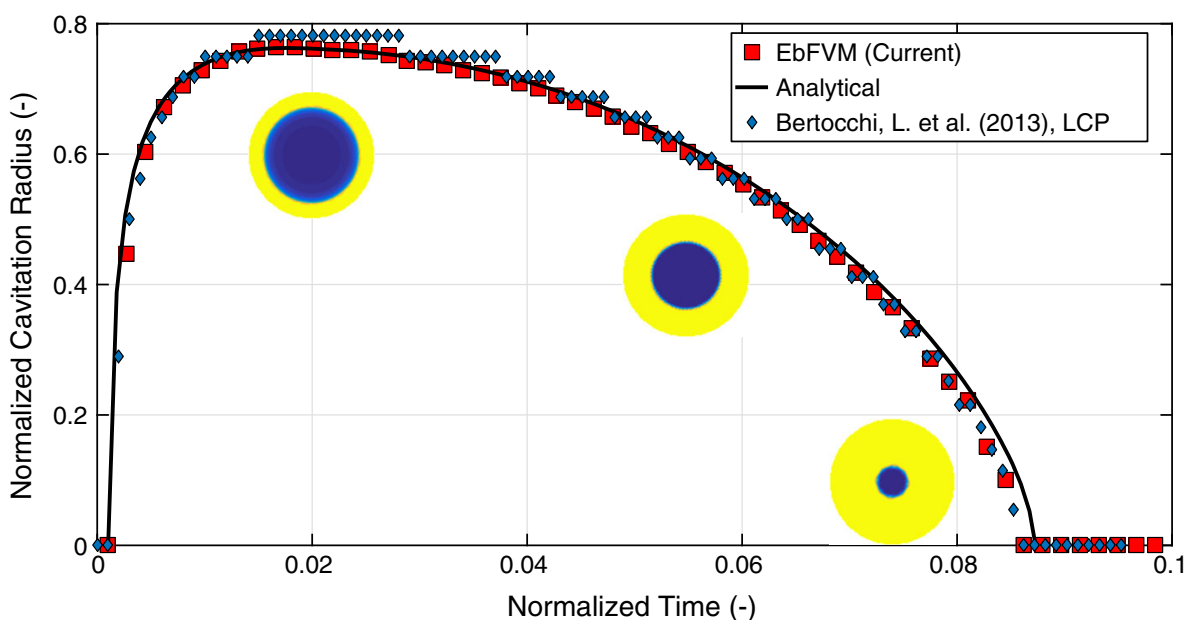


Table 4 General simulation parameters for the sliding bearing case with viscosity-pressure, viscosity-shear-thinning and density-pressure correction

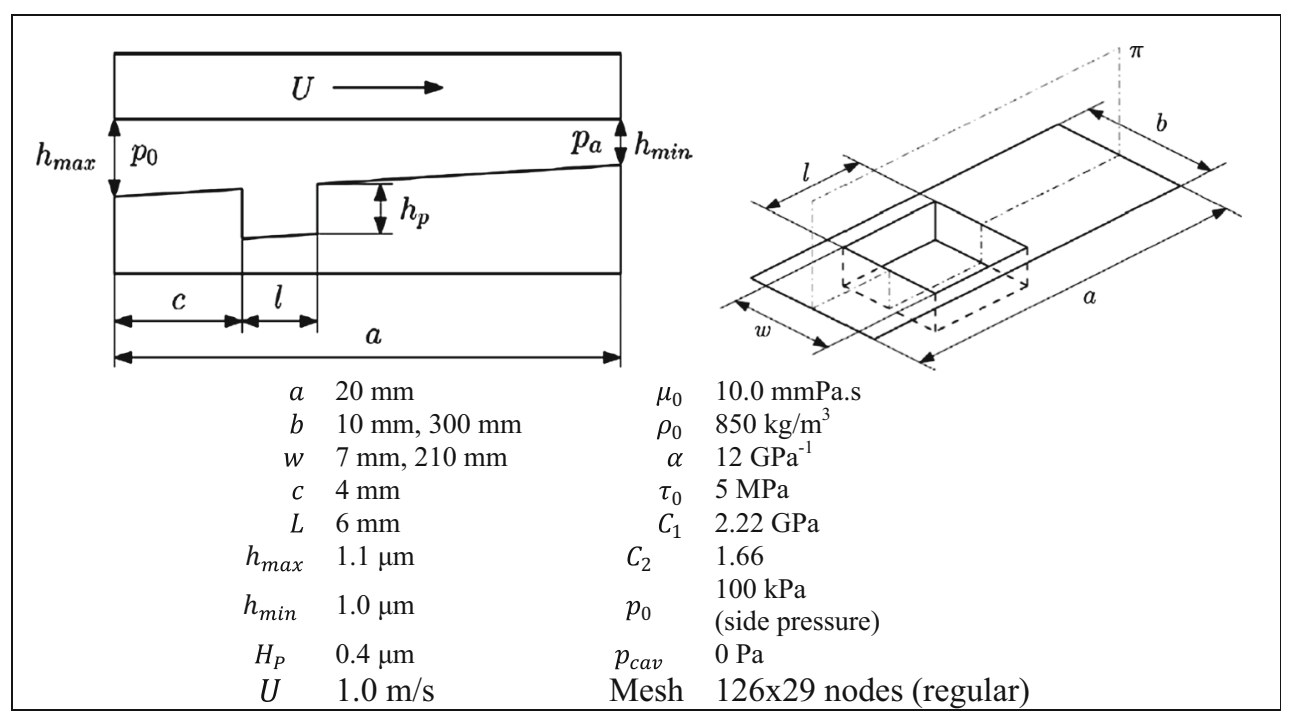

Fig. 6 Comparative results for the sliding bearing case with viscosity-pressure, viscosityshear-thinning and densitypressure correction

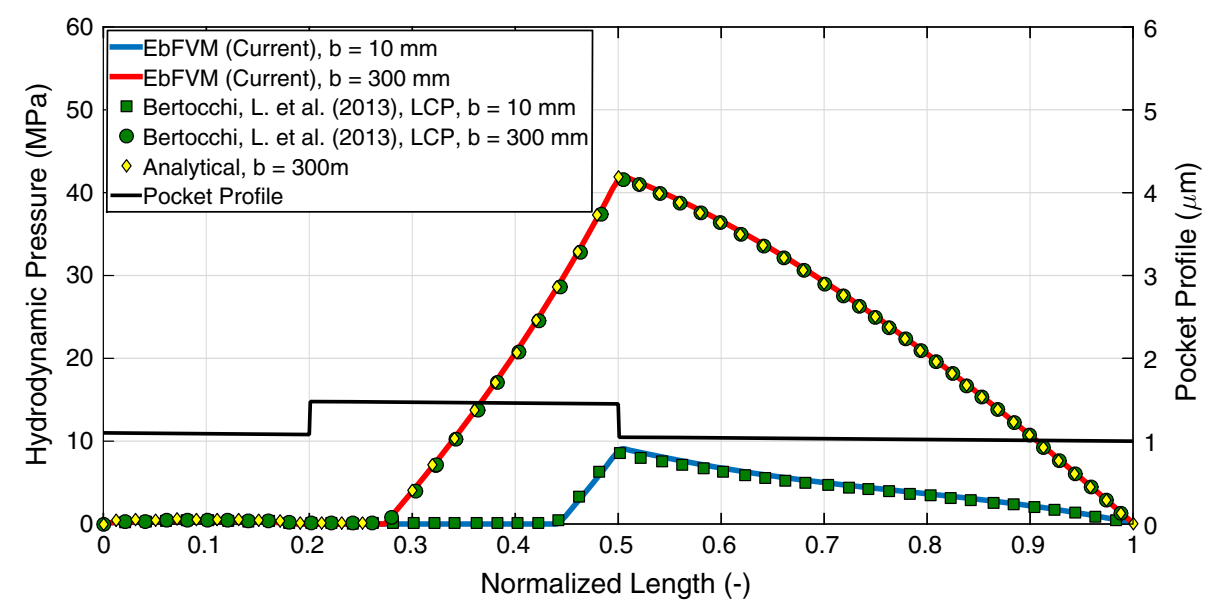

Table 5 Geometric and operational parameters for the textured sliding bearing simulations

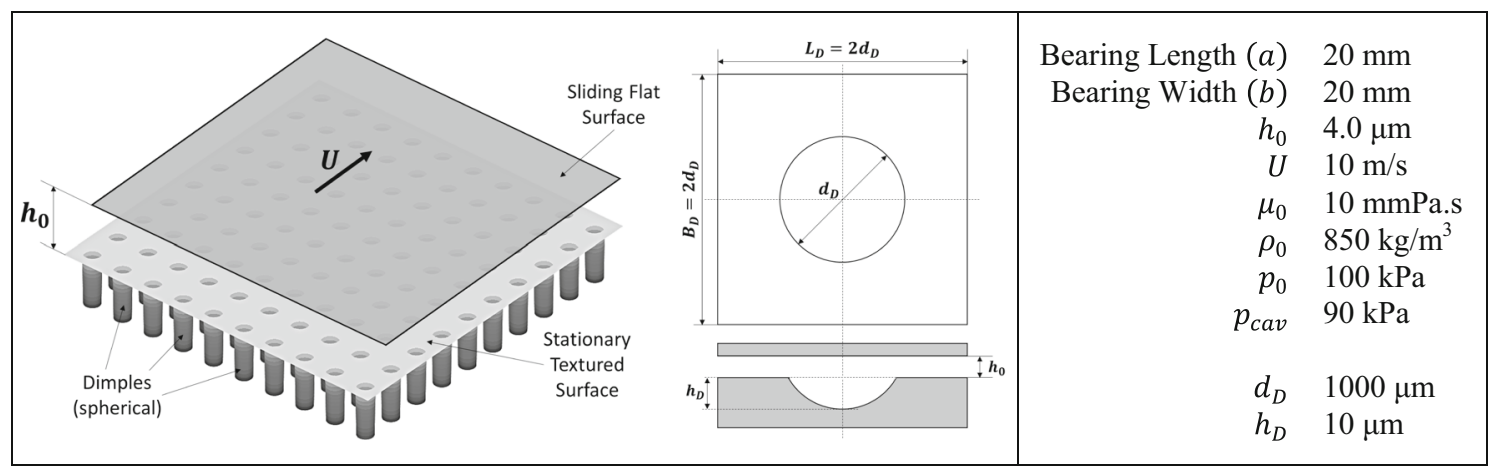

more pronounced. Such meshing control may well be used to reduce the number of nodes, and so the computational cost of simulations, needed to discretize the same textured pad when compared to structured grids. Figure 7 illustrates both the regular and irregular meshes of three representative texture variants with $10 \times 10,10 \times 6$ and $10 \times 2$ 

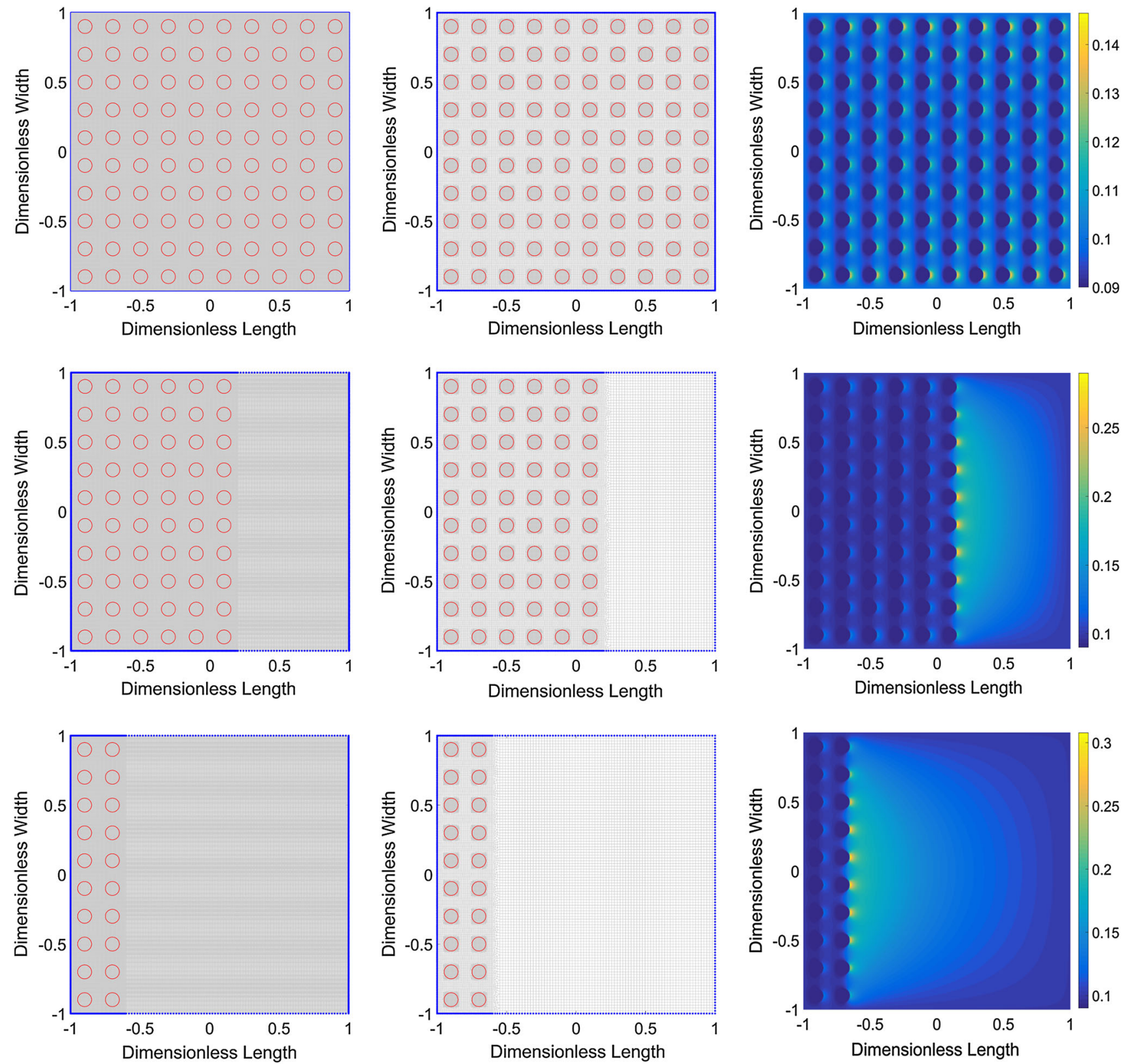

Fig. 7 Representative texture variants for the sliding bearing simulations and the associated hydrodynamic pressure results

dimples cells, as well as the associated hydrodynamic pressure fields calculated; notice the considerable decrease in the number of nodes for the unstructured meshes.

The number of outer iterations of the extended SOR algorithm (see Appendix 5), the computational time for convergence and the hydrodynamic load capacity are employed for comparing the results calculated on the regular and irregular meshes. The optimum relaxation factors of the SOR method for pressure $\left(\omega_{p}\right)$ have been found empirically by numerical experimentation. The optimum values for each texture pattern and mesh topology are displayed in Table 6; the relaxation value for the lubricant film fraction (cavitation) is assumed fixed as $\omega_{\theta}=1$. All the simulations were carried out in MATLAB in a computer with $8 \mathrm{~GB}$ RAM and Intel Core i7-3630 CPU $2.40 \mathrm{GHz}$; the algorithm reported in Appendix 5 is implemented as a MATLAB MEX-File that calls a compiled $\mathrm{C}$ function for performance improvement.

As can be verified in Table 6, the variation on the mesh topology hardly changed the overall load carrying capacity for all texture cases examined, but led to a significant speedup in the simulation time, yielded essentially by the decrease in the number of degrees of freedom (DOFs) for the irregular grids. Such occurrence demonstrates the 
Table 6 Performance of the EbFVM for different texture patterns and mesh topologies

\begin{tabular}{|c|c|c|c|c|c|c|c|c|c|c|}
\hline \multirow[t]{2}{*}{ Dimples } & \multicolumn{5}{|c|}{ Regular mesh } & \multicolumn{5}{|c|}{ Irregular mesh } \\
\hline & DOFs & $\omega_{p}$ & Iterations & Time (s) & $W(\mathrm{~N})$ & DOFs & $\omega_{p}$ & Iterations & Time (s) & $W(\mathrm{~N})$ \\
\hline $10 \times 10$ & 368,449 & 1.98 & 906 & 11.27 & 38.91 & 140,935 & 1.86 & 954 & 4.58 & 38.91 \\
\hline $10 \times 8$ & 307,142 & & 990 & 10.23 & 41.57 & 115,061 & 1.88 & 1572 & 6.18 & 41.60 \\
\hline $10 \times 6$ & 245,835 & & 1268 & 9.87 & 44.84 & 88,982 & 1.91 & 1867 & 5.74 & 44.91 \\
\hline $10 \times 4$ & 183,921 & & 1534 & 8.71 & 47.06 & 62,794 & 1.85 & 2579 & 5.05 & 47.15 \\
\hline $10 \times 2$ & 122,614 & & 1638 & 6.48 & 48.24 & 36,607 & 1.91 & 2092 & 2.14 & 48.35 \\
\hline
\end{tabular}
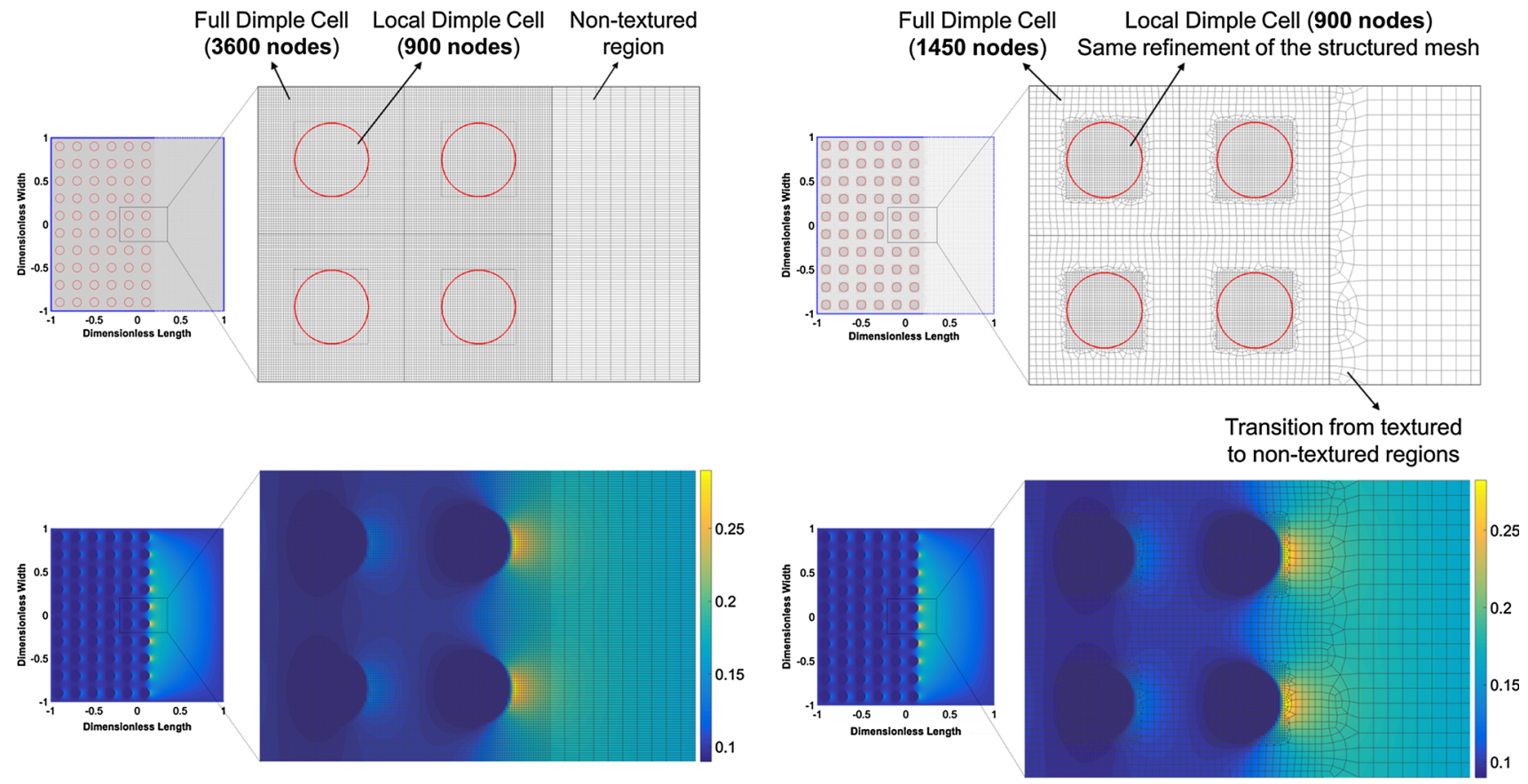

Full Dimple Cell Local Dimple Cell (900 nodes)

(1450 nodes) Same refinement of the structured mesh

Fig. 8 Detailed view of the hydrodynamic pressure distributions calculated in the regular (left) and irregular (right) meshes for the same texture variant

enhancements that can be achieved by using the current FVM; this is a result of the newly acquired capability of deploying non-structured meshes to simulate lubricated contact, which in turn can contribute either to reduce the number of DOFs in problems with complex geometries or to improve, by mesh refinements, the solution accuracy near regions characterized by special surface features in the contact domain. Although the absolute computational efficiency of the method is not the main concern at this point, since the idea of this numerical experiment is to illustrate how irregular meshes can be used to minimize the simulation time of lubrication problem with textured surfaces, the current formulation yields simulations times comparable to those reported for similar textured bearings in a recent publication [15].

We believe that performing a thorough and rigorous quantitative comparison between different solvers and methods in term of computational cost is a very important task. However, given the complexity of such task for extracting and quantifying the individual contribution to efficiency provided by cavitation algorithms, discretization schemes, hardware and code optimization methodologies followed by different researchers, this is deemed outside the scope of the present work and is the subject of the authors' current and future research efforts. At this stage, it may be worth mentioning that the most efficient solvers can potentially be obtained combining reliable discretization schemes, such as the one proposed here for unstructured grids, and efficient solution methods (see, e.g., [15]).

Finally, Fig. 8 depicts a detailed view of the hydrodynamic pressure distributions calculated on the regular and irregular meshes for the same texture variant. As expected, no noticeable difference is observed between the pressure fields. 
Table 7 Performance of the EbFVM for the $10 \times 6$ texture variant discretized locally with different grid densities

\begin{tabular}{lcccc}
\hline Nodes per dimple cell & Total DOFs & $\omega_{p}$ & Iterations & Time (s) \\
\hline $20 \times 20$ & 34,155 & 1.98 & 249 & 0.26 \\
$30 \times 30$ & 69,075 & & 443 & 0.98 \\
$40 \times 40$ & 115,995 & & 672 & 2.43 \\
$50 \times 50$ & 174,915 & & 925 & 5.66 \\
$60 \times 60$ & 245,835 & & 1268 & 9.87 \\
\hline
\end{tabular}

It is important to remark that the results displayed in Table 6, particularly the number of iterations, do not follow a monotonic pattern as the number of DOFs is reduced; this is somewhat counterintuitive but is solely due to the fact that each dimple variant used to generate the results reproduced in Fig. 7 corresponds to a different geometric domain. In fact, the monotonic pattern is expected be observed if the contact geometry (number of dimples rows) is kept constant and only the mesh topology is allowed to change. This has been here evaluated through a set of simulations for the $10 \times 6$ texture variant and regular meshes. In this case, each dimple cell was discretized locally by different grid densities, i.e., $20 \times 20,30 \times 30$, $40 \times 40,50 \times 50,60 \times 60$. The overall results are summarized in the Table 7. As can be seen, as the total number of mesh nodes increases, the amount of interaction required for convergence also increases accordingly.

\section{Conclusion}

This paper introduces EbFVM as a new paradigm in the simulation of lubrication problems characterized by thin fluid films in the presence of cavitation. The EbFVM algorithm developed to solve the conservative form of the equation of the mechanics of viscous thin films has been tested and successfully validated by comparison with existing alternative algorithms and full CFD simulations. The test cases presented have been used to demonstrate that the numerical approach proposed is robust and versatile and can incorporate compressibility, piezo-viscosity and shear-thinning of the lubricant, while being applicable to any transient hydrodynamic lubrication problem in the presence of cavitation. By successfully combining the flexibility of the FEM to deal with irregular geometries and the inherent conservative nature of the FVM, one of the main features of the EbFVM is that it allows the use of irregular meshes to discretize geometrically complex domain. This is shown to confer enormous flexibility to the method and enables a significant reduction in the computational requirements for solving lubrication problems in the presence of textured surfaces. Thus, the proposed method offers a new promising route for the improvement and design optimization of tribological surfaces.
Acknowledgments The authors gratefully acknowledge the partial support of the São Paulo Research Foundation (FAPESP) via the R\&D Consortium "TriboFlex"-PIT Project 2009/54891-8 and the Brazilian Federal Agency for the Support and Evaluation of Graduate Education (CAPES) - Scholarship Proc. BEX 2025/13-2 responsible for sponsoring the second phase of the $\mathrm{PhD}$ project. D.D. also acknowledges the support of the EPSRC Platform Grant EP/G026114/1. All simulation data is made freely available upon request to the following address tribology@ imperial.ac.uk.

Open Access This article is distributed under the terms of the Creative Commons Attribution 4.0 International License (http://crea tivecommons.org/licenses/by/4.0/), which permits unrestricted use, distribution, and reproduction in any medium, provided you give appropriate credit to the original author(s) and the source, provide a link to the Creative Commons license, and indicate if changes were made.

\section{Appendix 1: Elements for EbFVM Discretization}

In the following, all the geometric and tensor properties of triangular Fig. 9 and quadrangular Fig. 10 elements are presented for the completeness of the EbFVM derivation. The properties are shown with respect to the standard transformed domain (parametric elements).

\section{Linear Triangular Element}

- Coordinates of the element nodes

$$
P_{1}=(0,0) \quad P_{2}=(1,0) \quad P_{3}=(0,1)
$$

- Coordinates of the edges' midpoints

$$
m_{1}=\left(\frac{1}{2}, 0\right) \quad m_{2}=\left(\frac{1}{2}, \frac{1}{2}\right) \quad m_{3}=\left(0, \frac{1}{2}\right)
$$

- Coordinates of the integration points

$$
p I_{1}=\left(\frac{5}{12}, \frac{1}{6}\right) \quad p I_{2}=\left(\frac{5}{12}, \frac{5}{12}\right) \quad p I_{3}=\left(\frac{1}{6}, \frac{5}{12}\right)
$$

- Coordinates of the SVC's centroids

$$
G_{1}=\left(\frac{5}{24}, \frac{5}{24}\right) \quad G_{2}=\left(\frac{7}{12}, \frac{5}{24}\right) \quad G_{3}=\left(\frac{5}{24}, \frac{7}{12}\right)
$$

- Areas of the SVCs

$$
\begin{aligned}
A_{\mathrm{SVC}_{1}}= & \frac{1}{6} \operatorname{det}\left[\boldsymbol{J}\left(G_{1}\right)\right] \quad A_{\mathrm{SVC}_{2}}=\frac{1}{6} \operatorname{det}\left[\boldsymbol{J}\left(G_{2}\right)\right] \\
A_{\mathrm{SVC}_{3}} & =\frac{1}{6} \operatorname{det}\left[\boldsymbol{J}\left(G_{3}\right)\right]
\end{aligned}
$$

- Coordinates of the normal vectors (transformed domain) 
Fig. 9 Coordinate transformation for linear triangular elements
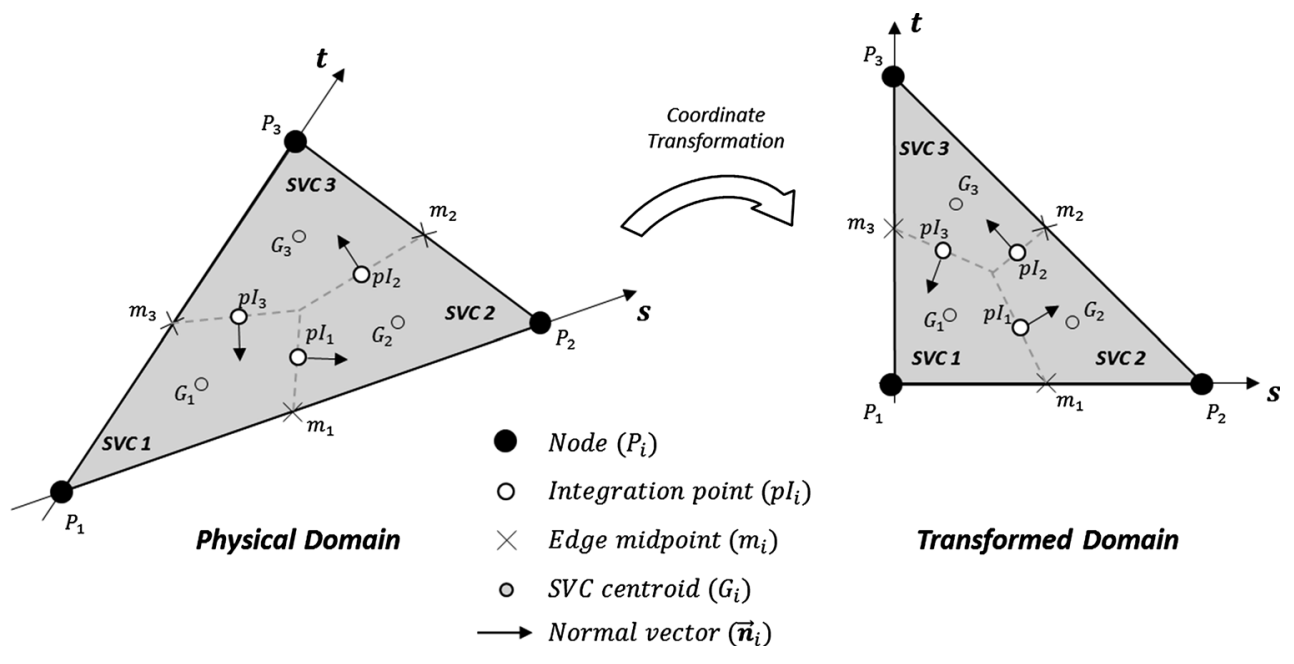

Transformed Domain

$$
\overrightarrow{\boldsymbol{n}}_{1}=\left(\frac{1}{6},-\frac{1}{3}\right) \quad \overrightarrow{\boldsymbol{n}}_{2}=\left(\frac{1}{6}, \frac{1}{6}\right) \quad \overrightarrow{\boldsymbol{n}}_{3}=\left(-\frac{1}{3}, \frac{1}{6}\right)
$$

- Coordinates of the normal vectors (physical domain) $\overrightarrow{\boldsymbol{n}}_{1}^{*}=\boldsymbol{R} \boldsymbol{J}\left(p I_{1}\right) \overrightarrow{\boldsymbol{n}}_{1} \quad \overrightarrow{\boldsymbol{n}}_{2}^{*}=\boldsymbol{R} \boldsymbol{J}\left(p I_{2}\right) \overrightarrow{\boldsymbol{n}}_{2} \quad \overrightarrow{\boldsymbol{n}}_{3}^{*}=\boldsymbol{R} \boldsymbol{J}\left(p I_{3}\right) \overrightarrow{\boldsymbol{n}}_{3}$

where the auxiliary matrix $\boldsymbol{R}$ is defined as:

$\boldsymbol{R}=\left[\begin{array}{cc}0 & -1 \\ 1 & 0\end{array}\right]$

- Interpolation and shape functions

$N_{1}(s, t)=(1-s-t) \quad N_{2}(s, t)=s \quad N_{3}(s, t)=t$

- Matrix for gradients interpolation

$$
\boldsymbol{B}(s, t)=\left[\begin{array}{ll}
\frac{\partial N_{1}(s, t)}{\partial s} & \frac{\partial N_{1}(s, t)}{\partial t} \\
\frac{\partial N_{2}(s, t)}{\partial s} & \frac{\partial N_{2}(s, t)}{\partial t} \\
\frac{\partial N_{3}(s, t)}{\partial s} & \frac{\partial N_{3}(s, t)}{\partial t}
\end{array}\right]=\left[\begin{array}{cc}
-1 & -1 \\
1 & 0 \\
0 & 1
\end{array}\right]
$$

- Jacobian matrix

$$
\boldsymbol{J}(s, t)=\boldsymbol{Z}_{e} \boldsymbol{B}(s, t)
$$

where $Z_{e}$ is the matrix with the global coordinates of the element nodes:

$\boldsymbol{Z}_{\boldsymbol{e}}=\left[\begin{array}{lll}x_{1} & x_{2} & x_{3} \\ y_{1} & y_{2} & y_{3}\end{array}\right]$

- Tensor for gradients' interpolation

$$
G_{j k}(s, t) \Rightarrow \boldsymbol{G}(s, t)=\boldsymbol{B}(s, t) \boldsymbol{J}(s, t)^{-1}
$$

- Tensor for diffusion calculation

$B_{m j k i s} \Rightarrow\left\{\begin{array}{l}B_{m j k i 1}=H_{m j k i}\left(p I_{1}\right)-H_{m j k i}\left(p I_{3}\right) \rightarrow \mathrm{SVC}_{1} \\ B_{m j k i 2}=H_{m j k i}\left(p I_{2}\right)-H_{m j k i}\left(p I_{1}\right) \rightarrow \mathrm{SVC}_{2} \\ B_{m j k i 3}=H_{m j k i}\left(p I_{3}\right)-H_{m j k i}\left(p I_{2}\right) \rightarrow \mathrm{SVC}_{3}\end{array}\right.$

where auxiliary tensor $H_{m j k i}$ is defined as:

$$
H_{m j k i}(s, t)=N_{m}(s, t) G_{j k}(s, t) n_{i}^{*}(s, t)
$$

- Matrices for the "flow-weighted" upwind scheme

$$
\begin{aligned}
\boldsymbol{A}^{\boldsymbol{s}} & =\left[\begin{array}{ccc}
0 & \left(1-\alpha_{p I_{1}}\right) & \alpha_{p I_{1}} \\
\alpha_{p I_{2}} & 0 & \left(1-\alpha_{p I_{2}}\right) \\
\left(1-\alpha_{p I_{3}}\right) & \alpha_{p I_{3}} & 0
\end{array}\right] \text { (29a) } \\
\boldsymbol{B}^{\boldsymbol{s}} & =\left[\begin{array}{ccc}
1 & -\left(1-\alpha_{p I_{1}}\right) \Lambda_{p I_{1}} & -\alpha_{p I_{1}} \Lambda_{p I_{1}} \\
-\alpha_{p I_{2}} \Lambda_{p I_{2}} & 1 & -\left(1-\alpha_{p I_{2}}\right) \Lambda_{p I_{2}} \\
-\left(1-\alpha_{p I_{3}}\right) \Lambda_{p I_{3}} & -\alpha_{p I_{3}} \Lambda_{p I_{3}} & 1
\end{array}\right]
\end{aligned}
$$

$$
\boldsymbol{C}^{s}=\left[\begin{array}{ccc}
\alpha_{p I_{1}}\left(1-\Lambda_{p I_{1}}\right) & \left(1-\alpha_{p I_{1}}\right)\left(1-\Lambda_{p I_{1}}\right) & 0 \\
0 & \alpha_{p I_{2}}\left(1-\Lambda_{p I_{2}}\right) & \left(1-\alpha_{p I_{2}}\right)\left(1-\Lambda_{p I_{2}}\right) \\
\left(1-\alpha_{p I_{3}}\right)\left(1-\Lambda_{p I_{3}}\right) & 0 & \alpha_{p l_{3}}\left(1-\Lambda_{p l_{3}}\right)
\end{array}\right]
$$

$\boldsymbol{D}^{s}=\left[\begin{array}{ccc}1 & 0 & -1 \\ -1 & 1 & 0 \\ 0 & -1 & 1\end{array}\right]$

where:

$$
\begin{gathered}
\alpha_{p I_{i}}= \begin{cases}1, & \text { if } q_{p I_{i}}>0 \\
0, & \text { if } q_{p I_{i}} \leq 0\end{cases} \\
\overrightarrow{\boldsymbol{\omega}}_{p \boldsymbol{I}}=\frac{\boldsymbol{A}^{s} \overrightarrow{\boldsymbol{q}}_{p \boldsymbol{I}}}{\overrightarrow{\boldsymbol{q}}_{\boldsymbol{p I}}} \vec{\Lambda}_{p I}=\max \left[\min \left(\overrightarrow{\boldsymbol{\omega}}_{\boldsymbol{p I}}, 1\right), 0\right]
\end{gathered}
$$


Fig. 10 Coordinate transformation for bilinear quadrangular elements
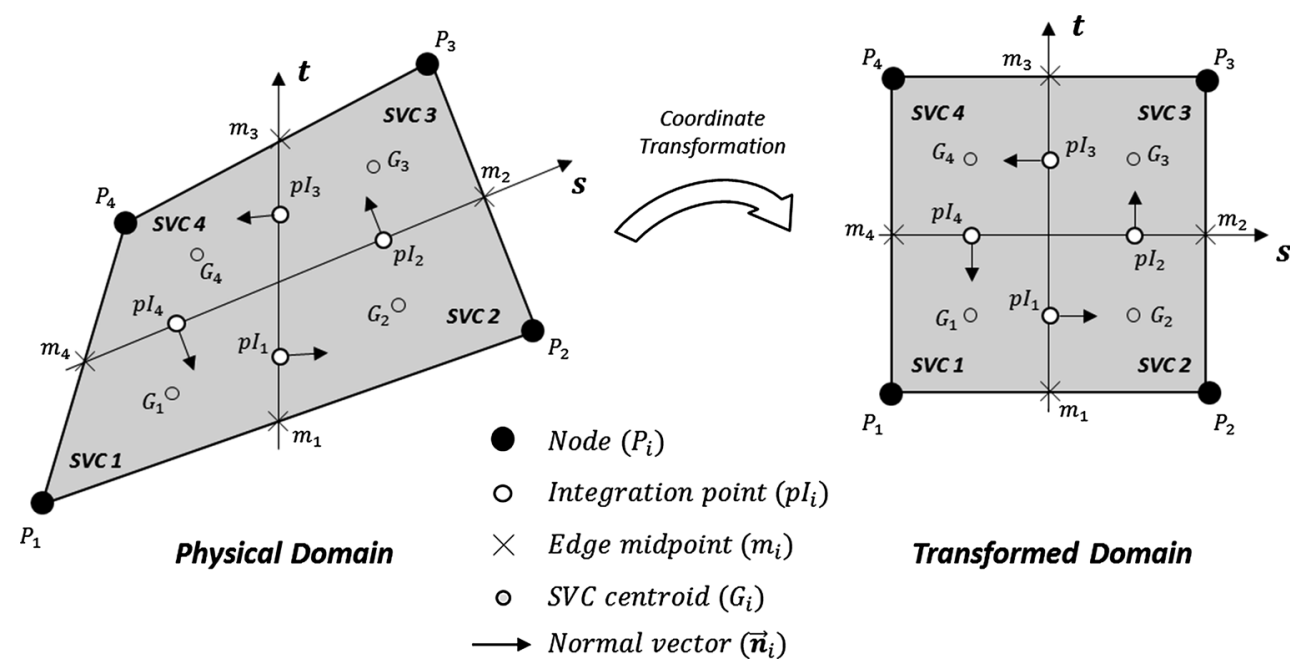

Transformed Domain

\section{Bilinear Quadrangular Element}

- Coordinates of the element nodes

$$
\begin{aligned}
& P_{1}=(-1,-1) \quad P_{2}=(1,-1) \quad P_{3}=(1,1) \\
& P_{4}=(-1,1)
\end{aligned}
$$

- Coordinates of the edge midpoints

$$
\begin{aligned}
& m_{1}=(0,-1) \quad m_{2}=(1,0) \quad m_{3}=(0,1) \\
& m_{4}=(-1,0)
\end{aligned}
$$

- Coordinates of the integration points

$$
\begin{gathered}
p I_{1}=\left(0,-\frac{1}{2}\right) \quad p I_{2}=\left(\frac{1}{2}, 0\right) \quad p I_{3}=\left(0, \frac{1}{2}\right) \\
p I_{4}=\left(-\frac{1}{2}, 0\right)
\end{gathered}
$$

- Coordinates of the SVC's centroids

$$
\begin{aligned}
G_{1} & =\left(-\frac{1}{2},-\frac{1}{2}\right) \quad G_{2}=\left(\frac{1}{2},-\frac{1}{2}\right) \quad G_{3}=\left(\frac{1}{2}, \frac{1}{2}\right) \\
G_{4} & =\left(-\frac{1}{2}, \frac{1}{2}\right)
\end{aligned}
$$

- Areas of the SVCs

$$
\begin{gathered}
A_{\mathrm{SVC}_{1}}=\operatorname{det}\left[\boldsymbol{J}\left(G_{1}\right)\right] \quad A_{\mathrm{SVC}_{2}}=\operatorname{det}\left[\boldsymbol{J}\left(G_{2}\right)\right] \\
A_{\mathrm{SVC}_{3}}=\operatorname{det}\left[\boldsymbol{J}\left(G_{3}\right)\right] \quad A_{\mathrm{SVC}_{4}}=\operatorname{det}\left[\boldsymbol{J}\left(G_{4}\right)\right]
\end{gathered}
$$

- Coordinates of the normal vectors (transformed domain)

$$
\begin{aligned}
& \overrightarrow{\boldsymbol{n}}_{1}=(0,-1) \quad \overrightarrow{\boldsymbol{n}}_{2}=(1,0) \quad \overrightarrow{\boldsymbol{n}}_{3}=(0,1) \\
& \quad \overrightarrow{\boldsymbol{n}}_{4}=(-1,0)
\end{aligned}
$$

- Coordinates of the normal vectors (physical domain) $\overrightarrow{\boldsymbol{n}}_{1}^{*}=\boldsymbol{R} \boldsymbol{J}\left(p I_{1}\right) \overrightarrow{\boldsymbol{n}}_{1} \quad \overrightarrow{\boldsymbol{n}}_{2}^{*}=\boldsymbol{R} \boldsymbol{J}\left(p I_{2}\right) \overrightarrow{\boldsymbol{n}}_{2} \quad \overrightarrow{\boldsymbol{n}}_{3}^{*}=\boldsymbol{R} \boldsymbol{J}\left(p I_{3}\right) \overrightarrow{\boldsymbol{n}}_{3}$ $\overrightarrow{\boldsymbol{n}}_{4}^{*}=\boldsymbol{R} \boldsymbol{J}\left(p I_{4}\right) \overrightarrow{\boldsymbol{n}}_{4}$

where the auxiliary matrix $\boldsymbol{R}$ is defined as:

$$
\boldsymbol{R}=\left[\begin{array}{cc}
0 & -1 \\
1 & 0
\end{array}\right]
$$

- Interpolation and shape functions

$$
\begin{array}{ll}
N_{1}(s, t)=\frac{1}{4}(1-s)(1-t) & N_{3}(s, t)=\frac{1}{4}(1+s)(1+t) \\
N_{2}(s, t)=\frac{1}{4}(1+s)(1-t) & N_{4}(s, t)=\frac{1}{4}(1-s)(1+t)
\end{array}
$$

- Matrix for gradients' interpolation

$$
\begin{aligned}
\boldsymbol{B}(s, t) & =\left[\begin{array}{ll}
\frac{\partial N_{1}(s, t)}{\partial s} & \frac{\partial N_{1}(s, t)}{\partial t} \\
\frac{\partial N_{2}(s, t)}{\partial s} & \frac{\partial N_{2}(s, t)}{\partial t} \\
\frac{\partial N_{3}(s, t)}{\partial s} & \frac{\partial N_{3}(s, t)}{\partial t} \\
\frac{\partial N_{4}(s, t)}{\partial s} & \frac{\partial N_{4}(s, t)}{\partial t}
\end{array}\right] \\
= & \frac{1}{4}\left[\begin{array}{cc}
(t-1) & (s-1) \\
(1-t) & (-1-t) \\
(1+t) & (1+s) \\
(-1-t) & (1-s)
\end{array}\right]
\end{aligned}
$$

- Jacobian matrix

$$
\boldsymbol{J}(s, t)=\boldsymbol{Z}_{e} \boldsymbol{B}(s, t)
$$

where $\boldsymbol{Z}_{\boldsymbol{e}}$ is the matrix with the global coordinates of the element nodes:

$$
\boldsymbol{Z}_{\boldsymbol{e}}=\left[\begin{array}{llll}
x_{1} & x_{2} & x_{3} & x_{4} \\
y_{1} & y_{2} & y_{3} & y_{4}
\end{array}\right]
$$

- Tensor for gradients interpolation

$$
G_{j k}(s, t) \Rightarrow \boldsymbol{G}(s, t)=\boldsymbol{B}(s, t) \boldsymbol{J}(s, t)^{-1}
$$

- Tensor for diffusion calculation 


$$
B_{m j k i s} \Rightarrow\left\{\begin{array}{l}
B_{m j k i 1}=H_{m j k i}\left(p I_{1}\right)-H_{m j k i}\left(p I_{4}\right) \rightarrow \mathrm{SVC}_{1} \\
B_{m j k i 2}=H_{m j k i}\left(p I_{2}\right)-H_{m j k i}\left(p I_{1}\right) \rightarrow \mathrm{SVC}_{2} \\
B_{m j k i 3}=H_{m j k i}\left(p I_{3}\right)-H_{m j k i}\left(p I_{2}\right) \rightarrow \mathrm{SVC}_{3} \\
B_{m j k i 4}=H_{m j k i}\left(p I_{4}\right)-H_{m j k i}\left(p I_{3}\right) \rightarrow \mathrm{SVC}_{4}
\end{array}\right.
$$

where auxiliary tensor $H_{m j k i}$ is defined as:

$$
H_{m j k i}(s, t)=N_{m}(s, t) G_{j k}(s, t) n_{i}^{*}(s, t)
$$

Matrices for the "flow-weighted" upwind scheme

$\boldsymbol{A}^{\boldsymbol{s}}=\left[\begin{array}{cccc}0 & \left(1-\alpha_{p I_{1}}\right) & 0 & \alpha_{p I_{1}} \\ \alpha_{p I_{2}} & 0 & \left(1-\alpha_{p I_{2}}\right) & 0 \\ 0 & \alpha_{p I_{3}} & 0 & \left(1-\alpha_{p I_{3}}\right) \\ \left(1-\alpha_{p I_{4}}\right) & 0 & \alpha_{p I_{4}} & 0\end{array}\right]$
- Approximation of the Diffusivity Matrix at the Integration Points

The diffusion matrix $\tilde{\boldsymbol{\Gamma}}^{p}(p I)$ may be written in tensor notation as:

$$
\tilde{\boldsymbol{\Gamma}}^{p}(p I) \Rightarrow \tilde{\Gamma}_{i j}^{p}(p I) \quad i, j=1,2
$$

Assuming that each component of the diffusivity matrix is approximated according to the same family of interpolation functions, then:

$\tilde{\Gamma}_{i j}^{p}(p I) \approx N_{m}(p I) \tilde{\Gamma}_{i j m}^{p} \quad m=1,2 \ldots, M$

where $\tilde{\Gamma}_{i j m}^{p}$ are the nodal values of $\tilde{\Gamma}_{i j}^{p}, M$ the number of nodes of the element and $N_{m}$ the respective interpolation functions (see Appendix 1).

- Approximation of the Pressure Gradients at the Integration Points

$$
\begin{aligned}
& \boldsymbol{B}^{\boldsymbol{s}}=\left[\begin{array}{cccc}
1 & -\left(1-\alpha_{p I_{1}}\right) \Lambda_{p I_{1}} & 0 & -\alpha_{p I_{1}} \Lambda_{p I_{1}} \\
-\alpha_{p I_{2}} \Lambda_{p I_{2}} & 1 & -\left(1-\alpha_{p I_{2}}\right) \Lambda_{p I_{2}} & 0 \\
0 & -\alpha_{p I_{3}} \Lambda_{p I_{3}} & 1 & -\left(1-\alpha_{p I_{3}}\right) \Lambda_{p I_{3}} \\
-\left(1-\alpha_{p I_{4}}\right) \Lambda_{p I_{4}} & 0 & -\alpha_{p I_{4}} \Lambda_{p I_{4}} & 1
\end{array}\right] \\
& \boldsymbol{C}^{s}=\left[\begin{array}{cccc}
\alpha_{p I_{1}}\left(1-\Lambda_{p I_{1}}\right) & \left(1-\alpha_{p I_{1}}\right)\left(1-\Lambda_{p I_{1}}\right) & 0 & 0 \\
0 & \alpha_{p I_{2}}\left(1-\Lambda_{p I_{2}}\right) & \left(1-\alpha_{p I_{2}}\right)\left(1-\Lambda_{p I_{2}}\right) & 0 \\
0 & 0 & \alpha_{p I_{3}}\left(1-\Lambda_{p I_{3}}\right) & \left(1-\alpha_{p I_{3}}\right)\left(1-\Lambda_{p I_{3}}\right) \\
\left(1-\alpha_{p I_{4}}\right)\left(1-\Lambda_{p I_{4}}\right) & 0 & 0 & \alpha_{p I_{4}}\left(1-\Lambda_{p I_{4}}\right)
\end{array}\right]
\end{aligned}
$$

$$
\boldsymbol{D}^{\mathbf{s}}=\left[\begin{array}{cccc}
1 & 0 & 0 & -1 \\
-1 & 1 & 0 & 0 \\
0 & -1 & 1 & 0 \\
0 & 0 & -1 & 1
\end{array}\right]
$$

where:

$$
\begin{gathered}
\alpha_{p I_{i}}=\left\{\begin{array}{l}
1, \quad \text { if } q_{c p I_{i}}>0 \\
0, \quad \text { if } q_{c p I_{i}} \leq 0
\end{array} \overrightarrow{\boldsymbol{\omega}}_{\boldsymbol{p} \boldsymbol{I}}=\frac{\boldsymbol{A}^{\boldsymbol{s}} \overrightarrow{\boldsymbol{q}}_{c \boldsymbol{I}}}{\overrightarrow{\boldsymbol{q}}_{\boldsymbol{c} \boldsymbol{I} \boldsymbol{I}}}\right. \\
\vec{\Lambda}_{p I}=\max \left[\min \left(\overrightarrow{\boldsymbol{\omega}}_{p \boldsymbol{I}}, 1\right), 0\right]
\end{gathered}
$$

\section{Appendix 2: Discretization of the Diffusion Term}

According to Eq. 10, it is possible to express the diffusive flux across each integration point $(p I)$ of a given element (e) as:

$d_{e}^{n}(p I)=\left[\tilde{\boldsymbol{\Gamma}}^{p}(p I) \nabla \tilde{p}(p I)\right]_{e}^{n} \cdot \overrightarrow{\boldsymbol{n}}_{e}^{*}(p I)$

where $p I=\left(s_{p I}, t_{p I}\right)$ denotes the coordinates of the integration points in the transformed domain. In the following deductions, the scripts $n$ and $e$ will be suppressed for shortness.
Pressure gradients $\nabla \tilde{p}(p I)$ may also be written in tensor notation as:

$\nabla \tilde{p}(p I) \Rightarrow \tilde{g}_{j}(p I)=\frac{\partial \tilde{p}(p I)}{\partial x_{j}} \quad j=1,2$

Assuming the elliptic characteristic of the diffusion term, the approximation of the pressure gradients can be accomplished by adopting linear interpolation based on the shape functions, yielding to the following tensor expression:

$\tilde{g}_{j}(p I) \approx G_{j k}(p I) \tilde{p}_{k} \quad k=1,2 \ldots, M$

where $\tilde{p}_{k}$ are the nodal pressures and $G_{j k}$ the interpolation tensor that correlates both pressure gradients approximations and element distortions (see Appendix 1).

Substituting Eq. 45 and Eq. 47 into Eq. 43, one obtains:

$$
\begin{aligned}
& d(p I)=H_{m j k i}(p I)\left(\tilde{\Gamma}_{i j m}^{p} \tilde{p}_{k}\right), \\
& H_{m j k i}(p I)=N_{m}(p I) G_{j k}(p I) n_{i}^{*}(p I)
\end{aligned}
$$

Since only geometric features are involved in $H_{m j k i}(p I)$, its value is constant for a fixed grid (non-adaptive mesh) 
and so can be calculated once in advance in a preprocessing step of the problem solution (see Appendix 1).

The total diffusive flux across every SCV $(s)$ that compound a given element is the sum of the respective fluxes computed for each of the two integration points located on its faces. Mathematically, it can be expressed as:

$d_{s}=P_{k s} \tilde{p}_{k}, \quad P_{k s}=B_{m j k i s} \tilde{\Gamma}_{i j m}^{p} \quad s, k=1,2 \ldots, M$

where $P_{k s}$ is defined as the diffusivity matrix and $B_{m j k i s}$ the geometric diffusion tensor of the element (see Appendix 1). Similarly to the $H_{m j k i}(p I)$ tensor, $B_{m j k i s}$ is also constant for fixed meshes and so can be calculated only once in a preprocessing procedure.

Finally, Eq. 49 can be written in matrix notation as:

$\overrightarrow{\boldsymbol{d}}_{e}^{n}=\boldsymbol{P}_{e}^{n}(\overrightarrow{\tilde{\boldsymbol{p}}})_{e}^{n}$

where the components of the vector $\overrightarrow{\boldsymbol{d}}_{e}^{n}$ correspond to the diffusive fluxes through each sub-control volume, $(\overrightarrow{\tilde{\boldsymbol{p}}})_{e}^{n}$ is the vector of the nodal pressures and $\boldsymbol{P}_{e}^{n}$ is the diffusivity matrix of the element.

\section{Appendix 3: Discretization of the Convective Term}

Analogously to the discretization of the diffusion term, the convective flux of Eq. 10 across each integration point $(p I)$ of a given element $(e)$ can be expressed as:

$c_{e}^{n}(p I)=[\tilde{q}(p I) \tilde{\theta}(p I)]_{e}^{n}, \quad \tilde{q}(p I)_{e}^{n}=\left[\tilde{\boldsymbol{\Gamma}}^{c}(p I) \tilde{\vec{v}}(p I)\right]_{e}^{n} \cdot \overrightarrow{\boldsymbol{n}}_{e}^{*}(p I)$

where $\tilde{q}(p I)$ is the local convective flux through the integration points $p I=\left(s_{p I}, t_{p I}\right)$. In the following derivations, the scripts $n$ and $e$ will be suppressed for shortness.

- Approximation of the Local Convective Flux at the Integration Points

Local convective flux $\tilde{q}(p I)$ may be written in tensor notation as:

$$
\begin{aligned}
\tilde{q}(p I) & =\tilde{C}_{i}(p I) n_{i}^{*}(p I), \quad \tilde{C}_{i}(p I)=\tilde{\Gamma}_{i j}^{c}(p I) v_{j}(p I) \\
i, j & =1,2
\end{aligned}
$$

Assuming that each component of $\tilde{C}_{i}(p I)$ is approximated according to the same family of interpolation functions, then:

$$
\tilde{C}_{i}(p I) \approx N_{m}(p I) \tilde{C}_{i m} \quad m=1,2 \ldots, M
$$

where $\tilde{C}_{i m}$ are the nodal values of $\tilde{C}_{i}, M$ the number of nodes of the element and $N_{m}$ the respective interpolation functions (see Appendix 1).

Substituting Eq. 53 into Eq. 52, one obtains:

$$
\begin{aligned}
& \tilde{q}(p I)=\tilde{Q}_{m i}(p I) \tilde{C}_{i m}, \quad \tilde{C}_{i m}=\left(\tilde{\Gamma}_{i j}^{c} v_{j}\right)_{m}, \\
& \tilde{Q}_{m i}(p I)=N_{m}(p I) n_{i}^{*}(p I)
\end{aligned}
$$

where $\tilde{Q}_{m i}(p I)$ is constant for a fixed grid (non-adaptive mesh) since only geometric features are involved.

- Approximation of the Film Fraction (Convective Variable) at the Integration Points

Approximation of the lubricant film fraction variable $(\tilde{\theta})$ is accomplished by using one of the most widespread advection scheme devised for EbFVM solutions, namely flow-weighted upwind scheme (FWUS) [29, 35]. This scheme is based on two important features: (1) the absolute preservation of the positivity of the coefficients of the discretized equations, which contributes for the stability of the numerical solution, and (2) the consideration of the local skewness of the flow, i.e., the local orientation of the fluid flow.

According to the FWUS scheme, the approximated value of the convective scalar variable at a given integration point depends only on the flow configuration in the immediate upstream sub-control volume. Considering the local indexation of nodes and faces in an element, one has [29]:

$\tilde{\theta}_{p I_{i}}= \begin{cases}\left(1-\Lambda_{p I_{i}}\right) \tilde{\theta}_{P_{i}}+\left(\Lambda_{p I_{i}}\right) \tilde{\theta}_{p I_{i+1}}, & \text { if } \tilde{q}_{p I_{i}}>0 \\ \left(1-\Lambda_{p I_{i}}\right) \tilde{\theta}_{P_{i-1}}+\left(\Lambda_{p I_{i}}\right) \tilde{\theta}_{p I_{i-1}}, & \text { if } \tilde{q}_{p I_{i}} \leq 0\end{cases}$

where the subscripts " $i$ " belong to the 4-cycle $\{1,2,3.4\}$ and are associated to each integration point $(p I)$ of the element. Thus, for instance, in a quadrangular element $k+1=1$ when $k=4$, whereas $k-1=$ 4 when $k=1$. In the above equation, $\Lambda_{p I_{i}}$ is the interpolation weighting factor that depends on the local flow orientation evaluated in agreement with the local flow ratio parameter $\omega_{p I_{i}}$, which in turn is defined as the ratio between the convective flux across the upwind face and the flow thorough the face where the associated integration point is located. Mathematically:

$$
\begin{gathered}
\omega_{p I_{i}}=\left\{\begin{array}{l}
\frac{\tilde{q}_{p I_{i+1}}}{\tilde{q}_{p I_{i}}}, \quad \text { if } \tilde{q}_{p I_{i}}>0 \\
\frac{\tilde{q}_{p I_{i-1}}}{\tilde{q}_{p I_{i}}}, \quad \text { if } \tilde{q}_{p I_{i}} \leq 0
\end{array}\right. \\
\Lambda_{p I_{i}}=\max \left[\min \left(\omega_{p I_{i}}, 1\right), 0\right]
\end{gathered}
$$

By assembling Eq. 56 for all integration points of the element, it is possible to define the following matrix expression that correlates $\tilde{\theta}_{i}$ to the nodal values $\tilde{\theta}_{P_{i}}$ : 
$\overrightarrow{\tilde{\boldsymbol{\theta}}}_{\boldsymbol{p} \boldsymbol{I}}=\underbrace{\left(\boldsymbol{B}^{\boldsymbol{s}^{-1}} \boldsymbol{C}^{s}\right)}_{\dot{U}} \overrightarrow{\tilde{\boldsymbol{\theta}}}=\boldsymbol{U} \overrightarrow{\tilde{\boldsymbol{\theta}}}$

where $\boldsymbol{B}^{\boldsymbol{S}}$ and $\boldsymbol{C}^{s}$ are $M \times M$ matrices calculated according to the local convective fluxes $\tilde{q}(p I)$ (see Appendix 1).

Finally, by substituting Eq. 54 and Eq. 57 into Eq. 51, the total convective flux across every SCV $(s)$ that compound a given element can be expressed as:

$c_{s}=C_{k s} \tilde{\theta}_{k}, \quad \boldsymbol{C}=\boldsymbol{D}[\operatorname{diag}(\overrightarrow{\tilde{\boldsymbol{q}}})] \boldsymbol{U} \quad s, k=1,2 \ldots, M$

where $\boldsymbol{D}$ is a $M \times M$ matrix that depends on the element type (see Appendix 1).

For simplicity, Eq. 58 can be written in matrix notation as:

$\overrightarrow{\boldsymbol{c}}_{\boldsymbol{e}}^{\boldsymbol{n}}=\boldsymbol{C}_{e}^{n}(\overrightarrow{\tilde{\boldsymbol{\theta}}})_{e}^{n}$

where the components of the vector $\overrightarrow{\boldsymbol{c}}_{e}^{n}$ correspond to the diffusive fluxes through each sub-control volume, $(\overrightarrow{\tilde{\boldsymbol{\theta}}})_{e}^{n}$ is the vector of the nodal pressures and $\boldsymbol{C}_{e}^{n}$ is the convective matrix of the element.

\section{Appendix 4: Discretization of the Source Terms}

According to Eq. 10, it possible to define two source terms associated to each $\operatorname{SCV}(s)$ of a given element $(e)$ :

$$
\begin{aligned}
f_{1 e, s}^{n} & =\left[\tilde{\theta}\left(\tilde{S}_{\mathrm{TS}}+\tilde{S}_{\mathrm{NS}}+\frac{\tilde{S}_{\mathrm{T}}}{\Delta t}\right)\right]_{P}^{n} \Delta \mathcal{D}_{s}^{e}, \\
f_{2 e, s}^{n} & =-\left[\tilde{\theta}^{n-1}\left(\frac{\tilde{S}_{\mathrm{T}}}{\Delta t}\right)^{n}\right]_{P} \Delta \mathcal{D}_{s}^{e} \quad s=1,2 \ldots, M
\end{aligned}
$$

where $\Delta \mathcal{D}_{s}^{e}$ is the physical area of the sub-control volume $s$. It is important to notice that both expressions in Eq. 60 are evaluated explicitly at the element nodes referred to the respective $\mathrm{SCV}$. By rearranging the terms of the equations in vector notation, one obtains:

$$
\begin{aligned}
& \left(\overrightarrow{\boldsymbol{f}}_{1}\right)_{e}^{n}=\left(\overrightarrow{\boldsymbol{F}}_{1}\right)_{e}^{n} \cdot(\tilde{\tilde{\boldsymbol{\theta}}})_{e}^{n}, \\
& \left(\overrightarrow{\boldsymbol{F}}_{1}\right)_{e}^{n} \Rightarrow F_{1 e, s}^{n}=\left[\left(\tilde{S}_{\mathrm{TS}}+\tilde{S}_{\mathrm{NS}}+\frac{\tilde{S}_{\mathrm{T}}}{\Delta t}\right)\right]_{P}^{n} \Delta \mathcal{D}_{s}^{e} \\
& \left(\overrightarrow{\boldsymbol{f}}_{2}\right)_{e}^{n}=\left(\overrightarrow{\boldsymbol{F}}_{2}\right)_{e}^{n} \cdot(\tilde{\tilde{\boldsymbol{\theta}}})_{e}^{n-1}, \\
& \left(\overrightarrow{\boldsymbol{F}}_{2}\right)_{e}^{n} \Rightarrow F_{2 e, s}^{n}=-\left[\left(\frac{\tilde{S}_{\mathrm{T}}}{\Delta t}\right)\right]_{P}^{n} \Delta \mathcal{D}_{s}^{e}
\end{aligned}
$$

where the components of $\left(\overrightarrow{\boldsymbol{f}}_{1}\right)_{e}^{n}$ and $\left(\overrightarrow{\boldsymbol{f}}_{2}\right)_{e}^{n}$ are the source terms associated to each element node, while $\left(\overrightarrow{\boldsymbol{F}}_{1}\right)_{e}^{n}$ and
$\left(\overrightarrow{\boldsymbol{F}}_{2}\right)_{e}^{n}$ are auxiliaries element vectors. Additionally, $(\overrightarrow{\tilde{\boldsymbol{\theta}}})_{e}^{n}$ and $(\tilde{\tilde{\boldsymbol{\theta}}})_{e}^{n-1}$ are the vectors of the nodal lubricant film fraction at the time instants $n$ and $(n-1)$, respectively; the operator "." denotes dot products.

\section{Appendix 5: Full Extended SOR Algorithm for the Solution of the Modified $p-\theta$ Reynolds Equation}

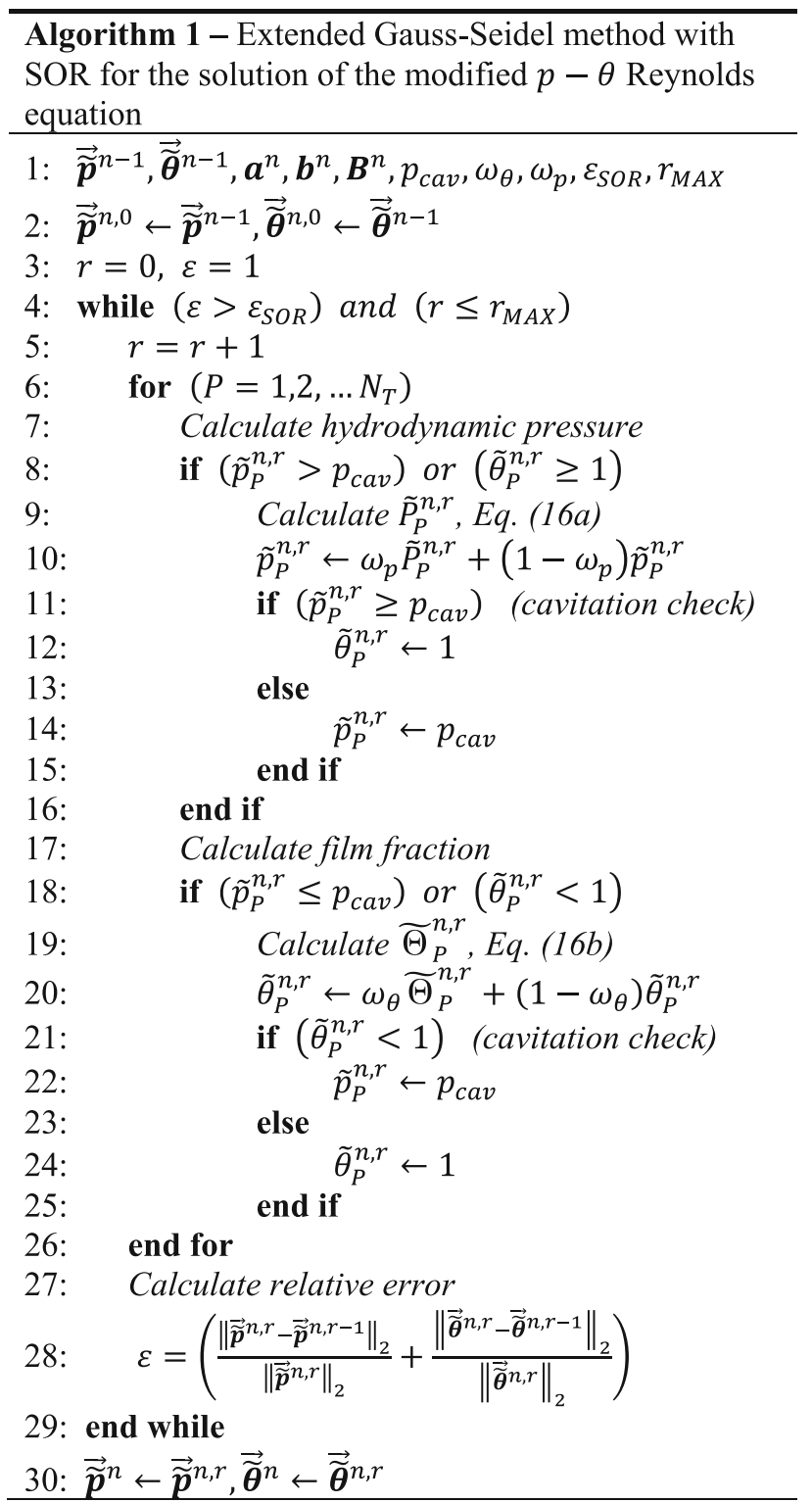




\section{References}

1. Boedo, S., Booker, J., Wilkie, M.: A mass conserving modal analysis for elastohydrodynamic lubrication. In: Lubricants and Lubrication: Proceedings of the 21th Leeds-Lyon Symposium on Tribology (1995)

2. Olson, E., Booker, J.: Hydrodynamic analysis of journal bearings with structural inertia and elasticity by a modal finite element method. In: Elastohydrodynamics-'96 Fundamentals and Applications in Lubrication and Traction: Proceedings of the 23rd Leeds-Lyon Symposium on Tribology held in the Institute of Tribology, Department of Mechanical Engineering (1997)

3. Optasanu, V., Bonneau, D.: Finite element mass-conserving cavitation algorithm in pure squeeze motion. Validation/application to a connecting-rod small-end bearing. Trans. ASME J. Tribol. 122(1), 162-169 (2000)

4. Booker, J.F., Boedo, S.: Finite element analysis of elastic engine bearing lubrication: theory. Revue Européenne des Éléments 10(6-7), 705-724 (2001)

5. Boedo, S., Booker, J.F.: Finite element analysis of elastic engine bearing lubrication: application. Revue Européenne des Éléments 10(6-7), 725-739 (2001)

6. Hajjam, M., Bonneau, D.: A transient finite element cavitation algorithm with application to radial lip seals. Tribol. Int. 40(8), 1258-1269 (2007)

7. Fatu, A., Hajjam, M., Bonneau, D.: A new model of thermoelastohydrodynamic lubrication in dynamically loaded journal bearings. Trans. ASME J. Tribol. 128(1), 85-95 (2006)

8. Jakobsson, B., Floberg, L.: The finite journal bearing considering vaporization. Trans. Chalmers University of Tech. Gothenburg 190 (1957)

9. Olsson, K.: Cavitation in dynamically loaded bearings. Trans. Chalmers University of Tech. Gothenburg 308 (1965)

10. Floberg, L.: Cavitation boundary conditions with regard to the number of streamers and tensile strength of the liquid. In: Cavitation and related phenomena in lubrication: proceedings of the 1st Leeds-Lyon Symposium on Tribology, held in the Institute of Tribology, Department of Mechanical Engineering, the University of Leeds, England (1974)

11. Elrod, H. G., Adams, M. L.: A computer program for cavitation and starvation problems. In Cavitation and Related Phenomena in Lubrication: Proceedings of the 1st Leeds-Lyon Symposium on Tribology, University of Leeds, Leeds, UK, Mechanical Engineering Publications Ltd, London, UK (1974)

12. Elrod, H.G.: A cavitation algorithm. Trans. ASME J. Lubr. Technol. 103(3), 350-354 (1981)

13. Giacopini, M., Fowell, M.T., Dini, D., Giacopini, M., Strozzi, A.: A mass-conserving complementary formulation to study lubricant films in the presence of cavitation. Trans. ASME J. Tribol. 132(4), 041702 (2010)

14. Bertocchi, L., Dini, D., Giacopini, M., Fowell, M.T., Baldini, A.: Fluid film lubrication in the presence of cavitation: a mass-conserving two-dimensional formulation for compressible, piezoviscous and non-Newtonian fluids. Tribol. Int. 67, 61-71 (2013)

15. Woloszynski, T., Podsiadlo, P., Stachowiak, G.W.: Efficient solution to the cavitation problem in fluid film lubrication. Tribol. Lett. 58 (2015)

16. Durany, J., Pereira, J., Varas, F.: A cell-vertex finite volume method for thermohydrodynamic problems in lubrication theory. Comput. Methods Appl. Mech. Eng. 195(44-47), 5949-5961 (2006)
17. Durany, J., Pereira, J., Varas, F.: Numerical solution to steady and transient problems in thermohydrodynamic lubrication using a combination of finite element, finite volume and boundary element methods. Finite Elem. Anal. Des. 44(11), 686-695 (2008)

18. Arghir, M., Alsayed, A., Nicolas, D.: The finite volume solution of the Reynolds equation of lubrication with film discontinuities. Intern. J. Mech. Sci. 44(10), 2119-2132 (2002)

19. Balcombe, R., Fowell, M.T., Olver, A.V., Ioannides, S., Dini, D.: A coupled approach for rolling contact fatigue cracks in the hydrodynamic lubrication regime: the importance of fluid/solid interactions. Wear 271(5-6), 720-733 (2011)

20. Ausas, R.F., Jai, M., Buscaglia, G.C.: A mass-conserving algorithm for dynamical lubrication problems with cavitation. Trans. ASME J. Tribol. 131(3), 031702 (2009)

21. Ausas, R.F., Jai, M., Ciuperca, I.S., Buscaglia, G.C.: Conservative one-dimensional finite volume discretization of a new cavitation model for piston-ring lubrication. Tribol. Int. 57, 54-66 (2013)

22. Raw, M. J.: A New Control Volume-Based Finite Element Procedure for the Numerical Solution of the Fluid Flow and Scalar Transport Equations. Ph.D. Thesis, University of Waterloo, Waterloo (1985)

23. Schneider, G.E., Raw, M.J.: Control volume finite-element method for heat transfer and fluid flow using colocated variables-1. Computational procedure. Numer. Heat Transf. 11, 363-390 (1987)

24. Frene, J., Nicolas, D., Degueurce, B., Berthe, D.: Hydrodynamic Lubrication: Bearings and Thrust Bearings. Tribology Series, vol. 33. Elsevier (1997)

25. Berthe, D., Godet, M.: A more general form of Reynolds equation-Application to rough surfaces. Wear 27, 345-357 (1974)

26. Spikes, H., Jie, Z.: History, origins and predictions of elastohydrodynamic friction. Tribol. Lett. 56, 1-25 (2014)

27. Stachowiak, G., Batchelor, A.: Engineering Tribology. Butterworth-Heinemann (2005)

28. Hurtado, F.S.V.: Three-Dimensional Finite Volume Formulation with Hybrid Unstructured Grids Applied to Reservoir Simulation. $\mathrm{Ph} . D$. Thesis, Federal University of Santa Catarina, Florianopolis, Brazil (2011). In Portuguese

29. Hurtado, F.S.V., Maliska, C.R.: A Family of positive flowweighted advection schemes for element-based finite-volume methods. Numer. Heat Transf. Part B Fundam. 62(2-3), 113-140 (2012)

30. Reddy, J.: An Introduction to the Finite Element Method. McGraw-Hill Series in Mechanical Engineering, McGraw-Hill Education, New York (2006)

31. Lohner, P.: Applied Computational Fluid Dynamics Techniques: An Introduction Based on Finite Element Methods. Wiley, New York (2008)

32. Ferziger, J., Peric, M.: Computational Methods for Fluid Dynamics. Springer, New York (2002)

33. Sahlin, F., Almqvist, A., Larsson, R., Glavatskih, S.: A cavitation algorithm for arbitrary lubricant compressibility. Tribol. Int. 40(8), 1294-1300 (2007)

34. Hartinger, M.: CFD Modelling of Elastohydrodynamic Lubrication. Ph.D. Thesis, Imperial College, London (2007)

35. Schneider, G.E., Raw, M.J.: A skewed, positive influence coefficient upwinding procedure for control-volume-based finite element convection-diffusion computation. Numer. Heat Transf. 9, $1-26(1986)$ 